\section{Cahiers de Narratologie}

Analyse et théorie narratives

$35 \mid 2019$

Le style comme événement

\title{
Réflexions sur l'histoire du changement stylistique : hypothèse événementielle, hypothèse variationniste et approche émergentiste
}

\section{Claire Badiou-Monferran}

\section{(2) OpenEdition}

Journals

Electronic version

URL: http://journals.openedition.org/narratologie/9558

DOI: $10.4000 /$ narratologie. 9558

ISSN: 1765-307X

Publisher

LIRCES

\section{Electronic reference}

Claire Badiou-Monferran, «Réflexions sur l'histoire du changement stylistique : hypothèse événementielle, hypothèse variationniste et approche émergentiste », Cahiers de Narratologie [Online], 35 | 2019, Online since 03 September 2019, connection on 15 November 2019. URL : http:// journals.openedition.org/narratologie/9558; DOI : 10.4000/narratologie.9558

This text was automatically generated on 15 November 2019.

Article L.111-1 du Code de la propriété intellectuelle. 


\title{
Réflexions sur l'histoire du
} changement stylistique : hypothèse événementielle, hypothèse variationniste et approche émergentiste

\author{
Claire Badiou-Monferran
}

Dans Yocaris (2016), le concept d' «événement de style ${ }^{1}$ » est prioritairement appliqué - quoique sans exclusive ${ }^{2}$ - à l'examen des « fictions postmodernes » :

Les fictions postmodernes se prêtent à une approche sémio-stylistique comme la nôtre [...] Elles exploitent de la manière la plus libre qui soit toutes les ressources de la prose littéraire [...] (Yocaris $2016: 25$ )

Sans préjuger de la validité de cette proposition, nous voudrions tester l'opérativité de l'approche événementielle pour les œuvres littéraires antérieures à la période contemporaine. Plus précisément, nous souhaiterions interroger, tout à la fois, le rendement et les limites de la notion "d'événement de style " pour l'histoire du changement stylistique ${ }^{3}$. Notre postulat de départ est que le concept interrogé n'a pas le même degré d'opérativité suivant les époques; qu'il est mieux à même de décrire l'histoire des changements stylistiques de la période dite « moderne ${ }^{4}$ » que de celle des changements stylistiques de l'ancien régime littéraire; que, pour la période de l'Early Modern $^{5}$, l'hypothèse variationniste du style s'avère sans doute plus productive que l'hypothèse événementielle; et que la reprise de l'une et de l'autre dans un cadre émergentiste - certes revisité - constitue un possible horizon d'attente pour la stylistique diachronique de ces prochaines décennies ${ }^{6}$. Nous illustrerons notre propos en invitant dans la réflexion l'observatoire des et « de relance ». 


\section{Quelques prérequis sur et de relance}

Sous cette appellation, la littérature critique désigne, à la suite de Serbat (1990), la configuration dans laquelle [e] et, succédant à l'oral à un intonème continuatif mineur ${ }^{7}$, et à l'écrit à un ponctuant pausal fort (le point, le point virgule, les deux points parfois, certaines virgules), articule deux actes de parole, dont le second prolonge et relance le premier :

(1) Michel. - regarde...maman...si c'était nécessaire je n'hésiterais pas... Madeleine. - tu hésiterais. Et tu aurais raison. Et c'est pourquoi je t'adore. (Cocteau, Les Parents terribles, 1938, II, $1: 235^{8}$ )

(2) $[\hat{A}]$ chaque coin de rue, des joueurs de luth et de cithare, des sages et des prophètes. Et aussi des lutteurs, des hâbleurs, des racoleurs de destins. Et sur chaque place, des chortens, des stupas [...] (Lanzmann, La horde d'or, 1994 : 344)

(3) Lui non plus ne peut ne pas se passer de nous. Et c'est comme ça, les familles. Il nous aime, à sa façon. Et même, il n'aime que nous. (Duhamel, Chronique des Pasquier, 2, $1934: 219)$

(4) Et mon père ajoutait : « il en est ici comme de la statue. Imaginerais-tu que pour le créateur il s'agisse de la description d'une bouche, d'un nez ou d'un menton? Non, certes. Mais du seul retentissement de tels objets les uns sur les autres, lequel retentissement sera par exemple douleur humaine. Et lequel par ailleurs il est possible de te faire entendre [...] (Saint-Exupéry, Citadelle, 1944 : 734)

Par opposition aux et "de clôture", connecteurs à part entière qui associent deux conjoints à l'intérieur d'un bloc sémantique (de type conclusif ${ }^{9}$, consécutif ${ }^{10}$, oppositif ${ }^{11}$, ou encore, final ${ }^{12}$ ) énonciativement fermé sur lui-même, les et de relance se distinguent, pour leur part :

(i) du point de vue énonciatif, par le caractère foncièrement suspensif (ie, continuatif) des enchaînements (non seulement phrastiques et/ou périodiques, mais aussi, possiblement propositionnels, et encore, syntagmatiques ${ }^{13}$ ) qu'ils instruisent ;

(ii) du point de vue lexical, par la possibilité de leur cooccurrence avec (a) les adverbiaux aussi, également, marquant l'adjonction (voir ci-dessus ex.2) (b) les adverbiaux même, en outre, plus, de plus, en plus [...] accompagnant un processus de surenchère (voir ci-dessus ex. 3) ; (c) ou encore avec les relatifs de liaison dont, lequel signalant une forme de contiguité ou de concomitance existentielle (voir ci-dessus ex. 4) ;

(iii) du point de vue structural, par leur caractère optionnel (ie, leur possibilité d'effacement: «A. Et B.» > «A. B.» - voir ci-dessus ex. 1-4) et leur propension à la démultiplication, suivant le schéma : «A. Et B. Et C. Et $\mathrm{D}$ [...]. Et $\mathrm{N}$ » (structure «en tresse » ou « en chapelet » dans la terminologie d'Aristote. Voir l'ex. 1 ci-dessus ${ }^{14}$ ).

Autrement dit, ils s'apparentent à des marqueurs pragmatiques (désormais $\mathrm{MP}^{15}$ ) : articulant des actes de parole sans lien implicatif, ils ne participent guère à la construction du sens discursif (si ce n'est pour signaler, en cooccurrence ou non avec des adverbiaux, un rapport lâche oscillant entre la concomitance existentielle, la succession temporelle et/ou la gradation argumentative - voir ci-dessus, ii). La liaison qu'ils établissent est de fait pleinement procédurale : à la production comme à la réception, les et de relance fonctionnent tout à la fois comme des signaux interactionnels de cohésion et de balisage - ie, de segmentation du discours (Capin \& Badiou-Monferran, à paraître). Ce type de marquage est déjà attesté en latin. Dans l'histoire au long cours du français, il intervient régulièrement, jusqu'en français préclassique, à différents niveaux (syntagmatique, propositionnel, phrastique et/ou 
périodique) en concurrence avec la virgule et le point médian ou final. Les travaux de Llamas Pombo (parmi d'autres, 2016 ${ }^{16}$ ) ont bien montré que, dans ce jeu de variables en compétition, et, en tant qu'« instruction de structure " vocalisable, consignant et/ou programmant un mode de profération des textes et des discours "à voix haute ", constituait le pendant oral d'une pratique de segmentation syntaxique que la ponctuation médiévale pouvait déjà par ailleurs prendre en charge pour le champ, purement visuel, de l'écrit et de sa lisibilité. Dans ce contexte, la cooccurrence, dans les manuscrits, d'un signe de ponctuation (généralement le point, plus rarement la virgule) et de et, à quelque niveau (syntagmatique, propositionnel, phrastique et ou périodique) que ce soit :

(5) Li reis marsilie out sun cunseil finet. // sin apelat clarin (...) de balaguet . // estamarin $Z$ eudropin sun per. // e priamun $Z$ guerlã le barbet. // e machiner $Z$ sun uncle maheu .// $Z$ iouner $Z$ malbien d'ultremer. // $Z$ blancadrins por la raisun cunter. (Roland, ca 1100, ms.Digby 23 Pt 2, fol. 2 v. « Après avoir tenu son conseil, le roi Marsile appela Clarin de Balaguer, Estamarin et Eudropin son pair, et Priamon, et Garlan le Barbu, et Machiner, et son oncle Mathieu, et Jouner, et Malbien d'Outremer, et Blancadrin, pour exposer sa décision ${ }^{17}{ }^{\prime}$. (Nous soulignons)

constituait alors une instruction de lecture bien particulière. Elle fonctionnait comme un indicateur «impressif» ou «emphatique» (Llamas Pombo 2016: 130), qui, introduisant de la gradation dans l'équivalence, hiérarchisait les conjoints en présentant le second comme argumentativement plus fort - ou informativement plus pertinent - que le premier (au même titre que la locution conjonctive Et même de l'ex. 4 ci-dessus $\left.{ }^{18}\right)$. Si ce dispositif textuel spécifique a disparu avec le passage de la tradition manuscrite à l'imprimé ${ }^{19}$, en revanche, l'emploi de MP de et comme démarcateur de syntagmes, de propositions, de phrase ou de périodes, en alternance (ou en cooccurrence) avec un signe de ponctuation, s'est maintenu jusqu'en français préclassique, dans le discours direct - à l'initiale d'un propos rapporté (ex. 6) et/ou en position médiane (ex.7 et 8 )-, dans le discours narrativisé (ex.9) et dans le récit (ex. 10-11) :

(6) Et [démarcateur de phrases et du discours rapporté à l'attaque de ce dernier] quoy, dit-il Diophanie, pour ce que je t'ay voulu tout donner [...] (Gombauld, L'Endimion, 1624, L. $3: 179$ )

(7) ô ! Pyzandre, que le destin est un puissant maistre ! Et [démarcateur de phrase à l'intérieur du DD] qu'en vain nostre raison se travaille, et [démarcateur de syntagme] s'efforce de luy resister, et [démarcateur de syntagme] de ne le suivre pas [...] (ibid. L. $1: 21$ )

(8) Quoy veux-tu que je vive, afin de faire voir toute la colere du ciel assemblée sur un seul homme ? Et [démarcateur de phrase en DD] ne m'empesches-tu de mourir une fois, qu'afin que je meure continuellement? Et [démarcateur de proposition subordonnée] que le souvenir des graces que tu m'as faites autres-fois me face consumer de regret [...]. (ibid., L.1:6)

(9) Je pris l'occasion d'en parler; et [démarcateur de proposition à la jonction du DN et du DI] leur dis que j'estimois ceste contrée heureuse d'estre en la garde d'une si grande deesse. Et en suite de cela [démarcateur de phrase relançant le DN], je me mis à leur representer son cours, ses mouvemens, les causes de ses diverses formes, tous ses changemens, et ses effects. (ibid., L. $4: 240$ ).

(10) Quand j'apperçeus quelques traces d'hommes, et [démarcateur de proposition en énonciation historique, dans le cadre d'une construction à cumul articulant une subordonnée à une proposition matrice] jugeay, selon l'apparence, que je n'estois plus exposé à nul danger. (ibid., L. $2: 132$ )

(11) Comme je m'entretenois de ces plaintes [...] j'entendis ouvrir la porte ; et [démarcateur de proposition non dépendante en énonciation historique] vis entrer 
un grand nombre de gens des plus notables [...] qui me regarderent fixement le visage [...] ; et puis [démarcateur de proposition subordonnée participiale 'en l'air', en construction détachée ${ }^{20}$ ] m'ayant interrogé sur mon pays, sur mon voyage, et sur l'accident qui m'estoit arrivé ; il se retirerent à part, et [démarcateur de proposition non dépendante] parlerent longtemps ensemble ; et [démarcateur de proposition non dépendante] à leurs gestes, il me sembloit qu'ils consentoient tous à une mesme chose (ibid., L. $4: 237$ ).

Le tableau ci-dessous permet de prendre la mesure de la relative bonne fréquence, à la fin du français préclassique, des Et de relance après un point, notamment dans les structures en [A. Et B. Et C...Et N], dites « en tresse » :

Tableau 1 Et de relance à la fin du français préclassique

\begin{tabular}{|c|c|c|c|}
\hline \multicolumn{4}{|c|}{$\begin{array}{l}\text { Gombauld, L'Endymion, } 1624 \text { : } 47978 \text { mots et } 973 \text { points. Tableau des } \\
\text { fréquences des enchaînements en «. Et ». }\end{array}$} \\
\hline \multicolumn{2}{|c|}{ EN DISCOURS } & \multicolumn{2}{|c|}{$\begin{array}{l}\text { EN RÉCIT } \\
\end{array}$} \\
\hline "En tresse » & «Isolé " & «En tresse » & «Isolé » \\
\hline \multicolumn{4}{|c|}{$\begin{array}{l}58 E t \text { de relance après un point } \\
\text { soit } 89 \% \text { des enchaînements en «. Et } \\
\text { et } \\
\text { des enchaînements réalisés après un point dans l'ensemble de } \\
\text { rage ( } 65 \text { occ. de } E t \text { après un point pour } 973 \text { points) }\end{array}$} \\
\hline \multirow[t]{2}{*}{4} & \multirow[t]{2}{*}{19} & $\begin{array}{r}35 \text { et de rel } \\
\text { soi }\end{array}$ & $\begin{array}{l}\text { ice en récit sur } 58 \text { et de } \\
\text { relance } \\
60,5 \% \text { du total }\end{array}$ \\
\hline & & 15 & 20 \\
\hline \multicolumn{4}{|c|}{$\begin{array}{l}7 \text { Et de clôture } \\
\text { soit }\end{array}$} \\
\hline & & & 4 \\
\hline
\end{tabular}

Il manifeste surtout la bonne implantation de ce type d'enchaînement en régime narratif : cette dernière y est même meilleure que dans les parties de discours rapporté. Or, après 1660, les enchaînements phrastiques par Et (de relance) connaissent une sérieuse baisse de fréquence à l'écrit, dans tous types de textes et dans tous types de configurations, mais tout particulièrement dans les segments d'énonciation historique des fictions narratives. Au tournant des XVII ${ }^{\mathrm{e}}$ et $\mathrm{XVIII}{ }^{\mathrm{e}}$ siècles, ils y sont devenus totalement résiduels (voir Badiou-Monferran 2018). Dans l'oral spontané, ils demeurent toujours productifs en revanche. Nombreuses sont ses attestations dans le français parlé contemporain :

(12) et à l'instant alors c'est du côté de Cannes qu'il y a eu une superbe occasion) ${ }^{\mathrm{s}}$

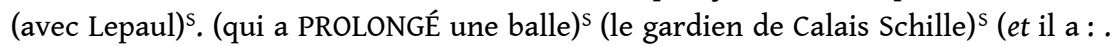
touché la balle sur la ligne) $)^{\mathrm{St}}$ (mais il a pas pu la: capter) ${ }^{\mathrm{S}}$ (il l'a simplement REpoussée) $)^{\mathrm{S}}$ (et au moment où : un autre euh joueur de Cannes Menaçait) ${ }^{\mathrm{S}}$ (eh bien il a PU : cette fois : capter la balle) ${ }^{\mathrm{S}}$ (GROS GROS GROS danger pour les joueurs de Calais) (alors qu'il reste deux ${ }^{\wedge}$ ou trois minutes à jouer) ${ }^{S+}$ (on est toujours à zéro

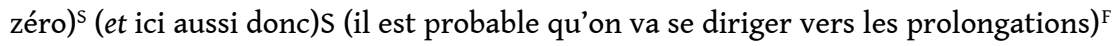
(Reportage radiophonique ${ }^{21}$ )

Ces données rendent compte du processus d'autonomisation de l'écrit qui, dans l'histoire au long cours du français, caractérise la période du français moderne (au sens large, conduisant du XVIe au XXIe siècle). À partir du français classique notamment, via la codification et la stabilisation des signes de ponctuation noire, le français écrit 
(littéraire et non littéraire) se dote d'un système de segmentation discursive autosuffisant, qui n'a plus besoin de faire appel aux marqueurs de segmentation de l'oral. Le MP et ne s'y maintient plus que dans les configurations ayant partie liée avec l'oralité, qu'il s'agisse :

(i) de fragments de discours rapportés (voir ci-dessus ex. 1-3 ou encore, ci-dessous, ex. 13)

(13) Depuis une semaine, elle m'a trouvé un nouveau truc : elle me met des points !; Y paraît qu'c'est la «méthode» du Docteur Guichenez - J'vous explique : quand j'fais un truc bien, j'ai des points et plus j'fais des trucs bien, plus j'ai de points......Et en fin d'année, j'ai un cadeau ! - Et oui ! (air content) (https://www.tabacologue.fr/ spip.php?article771)

(ii) du cas des intrusions d'auteur, à l'initiale d'une proposition incidente, où l'instance locutoire fait entendre sa voix

(14) Paul - et c'est fort heureux - a renoncé à son projet (Bally, Charles, Cahiers de F.

de Saussure, I, 40, 1941, cité par Antoine, 1958, p. 634)

(iii) de tous les cas où l'énonciateur s'implique dans son énoncé, pour en hiérarchiser les termes, comme dans les systèmes comparatifs corrélatifs (les structures en plus...et plus), au sein desquels et de relance marque un effet de gradation (ex. 15), ou dans les constructions averbales détachées où et extrait et met en relief l'unité de discours introduite par ses soins (ex. 16)

(15) plus il est chauve, et plus Thierry sourit (Blog des crados, 2006, http:// lescrados.canalblog.com/archives/p65-5.html)

(16) n'étant pas journalistes, ils vécurent heureux (et longtemps) (BETC, https:// betc.com/fr/socit/netant-pas-journalistes-ils-vecurent-heureux-et)

En dehors de ces configurations, dans les écrits non littéraires du moins, et de relance, notamment après un point, n'est plus (ou peu) attesté. Comment expliquer sa résurgence dans les fictions littéraires modernes, et notamment dans les séquences narratives de ces dernières?

\section{L'hypothèse " événementielle » à l'épreuve des fictions " modernes » : évolution(s) et variation(s) des emplois de et de relance en régime narratif littéraire à partir de 1850}

La saillance des et de relance dans le corpus des fictions narratives modernes n'est pas quantitative. Sans préjuger, il est vrai, de la part des et «de relance» et des et «de clôture » dans les suites en [A. Et B], nos enquêtes de voisinage sur Frantext attestent que ce tour, qui occupait le $1^{\text {er }}$ rang des enchaînements après un point dans le souscorpus des fictions narratives préclassiques, et le $4^{\mathrm{e}}$ dans celui de l'ensemble des productions écrites consignées dans la base pour la même période (1550-1650), recule en $6^{\mathrm{e}}$ position dans les romans modernes de la tranche 1850-1960, alors qu'il remonte à la $3^{\text {e }}$ place des enchaînements attestés pour le total des productions (romanesques et non romanesques) de la tranche 1850-1960. Ce chassé-croisé, qui s'articule autour d'une longue période de quasi-désattestation de la suite [A.Et B] à l'âge classique (période durant laquelle le tour n'occupe plus que la $17^{\mathrm{e}}$ position des enchaînements après un point attestés dans la base, et où il régresse même à la $19^{\mathrm{e}}$ place dans le corpus romanesque), laisse à penser que la résurgence des et de relance dans les fictions 
narratives modernes, commentée à l'envi par la critique auteuriste (parmi bien d'autres, Proust 1920 ; Thibaudet 1935), la stylistique de genre (Antoine 1962 ou encore Bordas 2005), et, plus récemment, les spécialistes d'histoire de la langue littéraire (Philippe 2013), ne constitue pas un phénomène massif, susceptible d'être mesuré, décrit ni glosé en termes quantitatifs : contrairement à ce qui avait pu être observé pour les fictions narratives préclassiques, dans les fictions narratives modernes, les fréquences absolues et relatives des et de relance se trouvent, globalement ${ }^{22}$, être bien plus basses que celles des autres productions contemporaines. Et c'est là que, pour mieux cerner l'avènement de ce qui sera perçu rétrospectivement comme un fait de style majeur, tant dans l'ordre des imaginaires collectifs qui construisent l'histoire de la langue littéraire qu'à l'échelle individuelle des signatures d'auteur, l'hypothèse événementielle, mobilisant des critères qualitatifs, peut s'avérer utile. De fait, dans l'histoire du changement stylistique, le retour du MP, de Flaubert à Duras (et même peut-être au-delà) semble bien pouvoir se laisser saisir, a minima, par les deux phénomènes de « rupture » et d' " intensification » constitutifs pour partie - du moins selon les coordinateurs de la présente livraison - de «l'événement de style ${ }^{23}$ ».

\section{Chaînes de stylisation et « rupture ». Première étape : du sémantique au sémiotique}

Anna Jaubert (2007) visualise le processus d'appropriation de la langue par le sujet parlant par une "diagonale du style ", au sein de laquelle interfèrent deux ordres de médiations : médiation des "genres de discours» (Jaubert 2007) et médiation des "imaginaires collectifs langagiers" (en d'autres termes: de la "langue littéraire", suivant les termes de Philippe \& Piat 2009). L'examen de la résurgence des et de relance dans les fictions narratives modernes mobilise conjointement l'une - le cadre du genre narratif - et l'autre - l'évolution des imaginaires opposant ancien et nouveau régime littéraire. Il doit en premier lieu interroger la manière dont la littérature narrative moderne, indépendamment de ses réalisations singulières (de Flaubert à Duras), quoiqu'à partir d'elles, a négocié sa propre relation à la langue commune. Nous formulons (sans originalité) l'hypothèse que, dans les exemples ci-dessous :

(17) Tous s'agenouillèrent. Il se fit un grand silence. Et les encensoirs, allant à pleine volée, glissaient sur leurs chaînettes (Flaubert, Trois contes, "Un cœur simple ", $1877: 72)$

(18) Ce Zizi, quelle grosse bête, de se causer tant de bile pour rien! Sur la tête de son enfant, elle n'aimait que son George. Et elle le baisait, et elle essuyait ses larmes (Zola, Nana, $1880: 1242$ )

(19) La lumière des après-midi de cet été-là Lol ne la voit pas. Elle, elle pénètre dans la lumière artificielle, prestigieuse, du bal de $\mathrm{T}$. Beach. Et dans cette enceinte largement ouverte à son seul regard, elle recommence le passé, elle l'ordonne, sa véritable demeure, elle la range. [...] Et cela recommence : À cet instant précis, une chose, mais laquelle? aurait dû être tentée qui ne l'a pas été. À cet instant précis, Lol se tient, déchirée, sans voix pour appeler à l'aide, sans argument, sans la preuve de l'inimportance du jour en face de cette nuit [...] Et cela recommence : les fenêtres fermées, scellées, le bal muré dans sa lumière nocturne les aurait contenus tous les trois et eux seuls. Lol en est sûre : ensemble ils auraient été sauvés de la venue d'un autre jour, d'un autre, au moins. (Duras, Le ravissement de Lol V. Stein, 1964 : 46-47)

le MP, dont le fonctionnement canonique de balise discursive est manifestement détourné, est en quelque sorte désémantisé et change de nature : il devient un signal sémiotique. Le fait est que si, en français moderne, le et de relance, comme on l'a vu, 
demeure productif à l'oral, où il continue de démarquer les unités de discours, il ne se maintient à l'écrit que dans quelques cas, ayant partie liée avec l'oralité (voir ci-dessus). Alors qu'il avait été préconisé en son temps par Vaugelas (1647), en toutes configurations :

" Au reste, on peut fort bien commencer une periode par la conjonction et, je dis mesme lors qu'il y a un point, qui ferme la periode precedente. Je n'en rapporteray point d'exemples, parce que tous nos bons Autheurs en sont pleins. Nous avons si peu de liaison pour les périodes qu'il ne faut pas nous oster celles-cy " (Vaugelas, Remarques sur la langue françoise, 1647, R. 399 : 637),

l'enchainement faisant succéder, à l'écrit, un $E t$ de relance à un point a acquis, deux cents ans plus tard, un statut marginal, infra-standard, comme en témoignent les discussions normatives qui lui sont consacrées dans Le Bon Usage (1988) ${ }^{24}$ :

«Des esprits logiciens considèrent comme une faute le fait de mettre une conjonction de coordination [notamment Et] après un point " (Grevisse, 1988 [1936], § $1032: 1566)$

C'est sans doute pourquoi Proust, commentant le «"style" de Flaubert » dans un texte célèbre paru en 1920, désigne comme irrégulier l'usage par ce dernier des Et de relance, mais en requalifiant la «faute " (selon la terminologie de Goosse ci-dessus) en «beauté grammaticale [n'ayant] rien à voir avec la correction »:

«En tous cas il y a une beauté grammaticale (comme il y a une beauté morale, dramatique, etc.), qui n'a rien à voir avec la correction. C'est d'une beauté de ce genre que Flaubert devait accoucher laborieusement. Sans doute cette beauté pouvait tenir parfois à la manière d'appliquer certaines règles de syntaxe [...] La conjonction "et" n'a nullement dans Flaubert l'objet que la grammaire lui assigne. Elle marque une pause dans une mesure rythmique et divise un tableau. En effet partout où on mettrait "et", Flaubert le supprime. C'est le modèle et la coupe de tant de phrases admirables. [...] En revanche là où personne n'aurait l'idée d'en user, Flaubert l'emploie. C'est comme l'indication qu'une autre partie du tableau commence, que la vague refluante, de nouveau, va se reformer. Tout à fait au hasard d'une mémoire qui a très mal fait ses choix : "La place du Carrousel avait un aspect tranquille. L'Hôtel de Nantes s'y dressait toujours solitairement; et les maisons par derrière, le dôme du Louvre en face, la longue galerie de bois, à droite, etc. étaient comme noyés dans la couleur grise de l'air, etc. tandis que, à l'autre bout de la place", etc. En un mot, chez Flaubert, "et" commence toujours une phrase secondaire et ne termine presque jamais une énumération » (Proust 1920).

"L'événement de style » ne tient pas ici à l'invention d'une forme nouvelle, non disponible dans la langue commune, mais à un processus de reconfiguration qui, jouant du réemploi de la forme en question (en l'occurrence, la conjonction et, en tant que marqueur, non de "clôture " mais de "relance») dans un régime textuel (celui de l'énonciation historique) qui n'en prévoyait pas (ou plus) l'utilisation, en opacifie, au plan sémantique, la lisibilité. Dans ce contexte, et ne fonctionne plus vraiment comme un MP démarquant tout en les liant les différentes unités de discours. Il se représente lui-même comme marque du discours oral, et comme contribuant, de ce fait, à l'oralisation de l'énonciation historique. Autrement dit, il constitue, tout à la fois, le support et la matérialisation de l'inscription d'une «sensibilité rectrice dans la prose, laissant entendre sa voix, par le rappel de la valeur phatique [des] connexions en tête de phrase » (Bordas 2005 : 27). Et en régime narratif littéraire est devenu un indicateur prosodique. Pour lors, du point de vue de l'histoire du changement stylistique, ce qui fait "événement " et constitue le "tournant radical ${ }^{25}$ " définissant le passage de l'ancien au nouveau régime littéraire des années 1850 , c'est bien le saut qualitatif 
faisant basculer, du point de vue de leur rapport à l'oralité, les fictions narratives modernes d'un régime sémantique à un régime sémiotique ; c'est cette transmutation d'une oralité constitutive en oralité montrée - ou, dans les termes de Philippe 2008, cette requalification des "appareils formels " de l'oralité, si caractéristiques des fictions narratives préclassiques et classiques, en "patron ${ }^{26} »$. Dans Philippe \& Piat 2009 , le changement de paradigme (au sens de Kuhn 2008) que constitue ce passage de l'écrit littéraire parlé à l'écrit littéraire vocalisée ${ }^{27}$ vient même servir de point de départ à la réflexion conduite par ailleurs sur les modalités langagières de l'autonomisation de la langue littéraire ${ }^{28}$.

Cette rupture majeure fera, comme le sait, l'objet d'inflexions et réinvestissements différents suivant les mouvements littéraires qui se succèderont. Dans la prose subjectiviste illustrée sous (17), le paradigme vocalisant s'infléchira dans le sens d'un « fond oratoire» (Thibaudet 1982: 238, cité dans Philippe \& Piat 2009:60), hérité des romantiques, et rendu ici sensible par le rythme anapestique (phrase courte, phrase courte, phrase longue), « relan[çant] la page ou le paragraphe par-delà [la] fatalité [de son] retombement " (Gracq 1995: 608); par l'insertion d'une expression détachée participiale, suspendant l'effet de relance en le dilatant; par le recours à l'imparfait, transformant l' " action » en « impression » (Proust, "À propos du style de Flaubert ») et " concrétisant ", du fait de son aspect sécant, «l'extension de la perception de la durée, et surtout de sa représentation" (Bordas $2005: 29)$; par la mobilisation, enfin, d'un et sémantiquement sous-déterminé, mais qui, en tant qu'item sémiotiquement surdéterminé, vecteur d'oralisation, grippe l'énonciation historique et en autorise, à partir de ce point de fuite, la relance.

En (18) supra, le paradigme vocalisant de la prose naturaliste s'éloigne du fond oratoire. Il se reconfigure dans une perspective socio-linguistique, invitant dans la prose littéraire tout un jeu de parlures populaires. Dans le prolongement du fragment de discours indirect libre qui précède, l'énonciation historique de la dernière phrase associe ses et oralisants à une certaine forme de dérapage lexical (du fait de l'ambiguïté de l'item " baisait ", faisant fusionner registre standard et registre trivial), à un schéma syntaxique des plus basiques (sujet-verbe-complément), et à un format répétitif (« [P1 en DDI], et P2, et P3») qui, jouant de la coordination polysyndétique en chaîne ouverte, mime la spontanéité d'un dire non concerté, non savant, improvisé dans la contemporanéité de son énonciation.

(19) illustre pour sa part le moment « discursif » du paradigme vocalisant : moment où la langue littéraire française se meut en laboratoire de «voix ». Les trois Et de relance qui s'y trouvent, du moins les deux derniers, signalent une "divergence énonciative » (au sens de Philippe $2000^{29}$ ) : articulant présent immédiat et passé récent, monde réel et monde virtuel, via le jeu des temps verbaux opposant dos à dos présents ponctuels et conditionnels passés à valeur d'irréel, ils inaugurent un espace transitionnel, constitué de l'hexasyllabe "Et cela recommence ", au sein duquel le démonstratif, ni totalement anaphorique, ni totalement déictique, fait communiquer voix narrative et voix de Lol, personnage "sans voix pour appeler à l'aide ", et qui, à défaut, se réfugie dans le monologue intérieur.

Du point de vue de l'histoire du changement stylistique, l'«événement de style " majeur qu'a pu constituer, autour des années 1850 , l'avènement du paradigme vocal succédant au paradigme parlé, s'est ainsi décliné en "une suite ininterrompue de [micro]-événements successifs » (Yocaris 2016: 25), définissant autant de moments 
(parmi d'autres, le moment oratoire, le moment socio-linguistique, le moment discursif ${ }^{30}$ ) qui en autorisaient la relance. Surgit du même coup un autre trait définitoire de l' "événement de style »: celui de sa dynamique fondamentalement actualisante, qui le protège de l'usure et d'une éventuelle hypostase en « fait de style ».

\section{Chaînes de stylisation et intensification. Deuxième étape : sur- sémiotisation et signifiance}

Si, dans la perspective diachronique que nous adoptons ici, le critère de la rupture (ou du saut qualitatif, requalifiant le plan sémantique en plan sémiotique) éclaire la nature des liens entre langue littéraire des fictions narratives modernes d'une part et langue commune contemporaine de l'autre, c'est bien plutôt le critère de l'intensification (à l'intérieur du plan sémiotique) qui semble opératoire pour décrire les relations dialectiques associant un état donné de la langue littéraire aux styles d'auteur qui, tout à la fois, le composent et en émanent.

\section{Sursémiotisation}

Revenons au moment «impressionniste» de la prose narrative française et à son versant flaubertien. Celui-là s'est exprimé dans celui-ci par la mise en faisceau de trois traits de déterminations langagières (les imparfaits de narration, le rythme ternaire, les et de relance : voir ci-dessus, ex. 17) définissant sa signature. A ce niveau, proprement auctorial, la prégnance de la petite musique flaubertienne ne se mesure nullement en termes de saut qualitatif, de rupture, de changement de plan, mais, d'un point de vue strictement quantitatif, à la régularité des associations internes au faisceau décrit cidessus - l'apparition en discours de l'un des trois traits de détermination langagière appelant celle des deux autres et réciproquement. Dans Un cœur simple, les quatre occurrences de $E t$ de relance après un point sont ainsi systématiquement adossées, dans $100 \%$ des cas, à celle de l'imparfait :

(20) Et jamais elle ne parlait de ses inquiétudes (Flaubert, Un cour simple, 1877 : 35)

Et Félicité priait en regardant l'image [...] (ibid. : 63)

Les persiennes n'ouvraient plus. Bien des années se passèrent. Et la maison ne se louait pas, et ne se vendait pas (ibid. : 67)

Tous s'agenouillèrent. Il se fit un grand silence. Et les encensoirs, allant à pleine volée, glissaient sur leurs chaînettes (ibid. : 72)

et dans $50 \%$ à un effet de rythme ternaire avec anapeste (voir ci-dessus les deux derniers extraits, enchainant phrase courte, phrase courte et phrase longue). L'intensité du phénomène associatif se mesure ainsi, quantitativement, à la récurrence des appariements attendus, et lorsque leur fréquence oscille, comme ici, entre $50 \%$ et $100 \%$, l'association se sur-sémiotise, en se donnant à lire non plus seulement comme une manifestation parmi d'autres $\mathrm{du}$ « fond oratoire » de la langue narrative moderne (celle, impressionniste, des années 1850), mais, par-delà, comme une signature auctoriale. Les pastiches cocteauesque et perecquien de la prose flaubertienne, conjuguant rythme anapestique, imparfaits et et de relance :

(21) Elle devenait aussitôt fort digne, harponnait quelque journal, et, dissimulée derrière les grandes feuilles ouvertes, en commençait la minutieuse lecture (Cocteau, Les Enfants terribles, Pléiade $2006: 595^{31}$ )

(22) Alors, ils se sentaient les maîtres du monde. Ils ressentaient une exaltation inconnue, comme s'ils avaient été détenteurs de secrets fabuleux, de forces 
inexprimables. Et, se donnant la main, ils se mettaient à courir, ou jouaient à la marelle, ou couraient à cloche pied le long des trottoirs et hurlaient à l'unisson les grands airs de Cosi fan tutte ou de la Messe en si (Perec, Les choses. Une histoire des années soixante. Press Pocket [1965] $1984: 64^{32}$ )

ou, à défaut, ses parodies zoliennes, qui en défigurent la physionomie en ne retenant du faisceau à trois éléments qu'un seul terme (en l'occurrence, et de relance) :

(23) [...] et ils se promenèrent sous les arbres, les bras à la taille, et ils allèrent se coucher dans l'herbe, où la rosée les trempa. (Zola, Nana, Paris, Flammarion, 2000 $\left.\left[{ }^{1} 1880\right]: 208^{33}\right)$

permettent de prendre toute la mesure du phénomène. Dans le premier cas, le processus de sursémiotisation est surjoué ; dans le second, il est déjoué. Pour autant, à chaque fois, du pastiche à la parodie, des marqueurs auctoriaux sont re-présentés comme étant représentatifs d'un style particulier. Il s'agit donc d'une représentation au carré, d'une représentation redoublée. La dynamique d'intensification s'en trouve dédoublée et "l'événement de style ", jouant de la superposition de trois paliers, celui, sémiotique, de la langue littéraire, celui sursémiotisant, des styles d'auteur et celui, apparenté à une sursémiotisation de second degré, de l'imitation ou de la reprise parodique de ces derniers - gagne là encore à être décrit comme «une suite ininterrompue d'événements », cette fois non pas tant « successifs » que " simultanés » (Yocaris 2016 : 25). La trajectoire de "l'événement de style » s'exerce donc, d'une part, horizontalement et dans la durée, à la faveur de sauts qualitatifs (de ruptures) entre langue commune (relevant du plan sémantique) et langue littéraire (relevant du plan sémiotique), et verticalement, de manière simultanée, selon un processus d'intensification caractérisant, au sein d'un ordre homogène (l'ordre sémiotique), la relation de la langue littéraire, à un moment donné de son histoire, avec les styles d'auteur qui l'alimentent et qu'elle génère, et encore, à un pallier supérieur, avec leur imitation et/ou reprise parodique (contemporaine ou chronologiquement décalée).

\section{Signifiance}

Dans certains cas, le mouvement d'intensification associé à l'avènement des styles d'auteur peut s'accompagner d'une rupture, ie d'un changement de plan lorsqu'il s'apparente, non pas à un processus de sursémiotisation mais à un processus de transcatégorisation, reconfigurant la matérialité sémiotique en matérialité « signifiante ». Sous cette appellation, et, plus précisément, sous celle de « signifiance », Meschonnic (2009) définit une expérience "anti-sémiotique », celle du rythme qui signifie sans générer la dualité du signe: «[p]ris dans la paradigmatique et la syntagmatique d'un discours, le rythme sens et sujet fait une sémantique généralisée, fonction de l'ensemble des signifiants, qui est la signifiance » (ibid. : 72). Autrement dit, « le signe est remplacé par le signifiant, mais celui-ci n'oppose pas le langage à la forme: "Il n'y a plus la double articulation du langage" (Meschonnic 1999: 118)» (Cordingley 2014 : 27-40). Le sens du rythme n'est pas extérieur au rythme. Le rythme n'indexe ni une pratique rédactionnelle historiquement circonscrite (parmi d'autres, celle du moment "oratoire » du paradigme vocalisant), ni une signature auctoriale (parmi d'autres, celle de Flaubert). Quittant le niveau sémiotique et faisant retour sur lui-même, il ne signifie rien d'autre que sa propre pulsation. C'est cette expérience chrono-graphique, éminemment subjective, singulière, unique - réfractaire, donc, à l'ordre rhétorique (par définition collectif), que Meschonnic dénomme " oralité » ${ }^{34}$, et que selon lui la littérature est en mesure, plus que tout autre production écrite, de 
délivrer : «La littérature est la réalisation maximale de l'oralité » (Meschonnic 1999: 117). Rien n'empêche, au demeurant, qu'oralité et signature auctoriale viennent à communiquer, à se rejoindre, à se superposer. C'est le processus qu'Apollinaire désignait sous l'appellation de "prosodies personnelles $»^{35}$ et que Cordingley (2014: 47-60), paraphrasant et commentant Meschonnic, définit en termes de «sémantique personnelle ». Pour autant, dans un poème comme

(24) Et l'unique cordeau des trompettes marines (Apollinaire, «Chantre», Alcools, 1913: 63)

c'est moins la petite musique d'Apollinaire qu'il nous est donné d'éprouver, avec ce et de relance, qui a fait couler beaucoup d'encre ${ }^{36}$, que l'expérience du rythme en actes, du rythme pur. N'embrayant sur aucun avant-texte, le petit mot est bien évidemment désémantisé, et ne fonctionne pas comme un MP (ie, comme un démarcateur d'unité de discours). Peu sollicité par ailleurs dans l'œuvre d'Apollinaire, et tout particulièrement dans Alcools, il ne contribue pas une marque de reconnaissance auctoriale et n'acquiert pas le statut, sémiotique, d'indice idiolectal. Et vient combler ici une lacune prosodique, au strict plan de la «signifiance ». Sa présence rend le propos du poète à l'excellence d'un alexandrin, dont la pulsation est immédiatement perceptible, pour qui le vocalise. Son « oralité » (au sens de Meschonnic) est constitutive. Elle existe indépendamment de son rattachement à un quelconque projet auctorial. Si celui-ci en vient à rencontrer celle-là (comme sans doute chez Flaubert) ${ }^{37}$, cette rencontre est fortuite, non prévisible.

Les «événements » qui se jouent au niveau de la «signifiance »-ie, les événements d'« oralité » selon Meschonnic - font intervenir, tout à la fois, le critère de la «rupture"-au sens où ils sollicitent un autre plan que les plans sémantiques et sémiotiques - et celui de l'intensification - au sens où l'expérience prosodique est perçue dans toute sa pureté, en elle-même et pour elle-même, indépendamment des enjeux sémantiques et sémiotiques qui, par ailleurs, la conditionnent. Assez logiquement, ce type d'événement, certes capital dans l'expérience des acteurs qui le vivent (son sujet producteur et ses récepteurs), qui se réalise en tous lieux, en toutes langues et à toutes les époques - Meschonnic (1999) cite Rabelais, Joyce, Gogol, Kafka... - et que la stylistique auteuriste se donne avec raison pour objet de décrire, n'intéresse guère l'histoire du changement stylistique, car, en tant qu'événement de discours singulier, unique, évanescent, il n'est pas historicisable. Nous laisserons donc les « événements de signifiance » de côté, pour interroger le rendement des opérations de sémiotisation (articulant langue commune et langue littéraire) et de sursémiotisation (articulant langue littéraire et styles d'auteur) pour la période de l'Early Modern.

\section{Early Modern : de l'hypothèse événementielle à I'hypothèse variationniste}

Nous partirons de Rabelais et de son Pantagruel qui, du moins dans l'édition princeps de 1532, fourmille de et de relance ${ }^{38}$. La grille événementielle permet-elle d'appréhender ces derniers? 


\section{Limites de l'hypothèse événementielle}

Quantitativement, l'emploi de relances est bien plus massivement sollicité chez Rabelais que chez Flaubert, Zola ou Duras. Dans les deux extraits suivants, sélectionnés à titre de sondage :

(25) Panurge commenca a estre en reputation en la ville de Paris par ceste disputation que il obtint contre l'Angloys/ \& faisoit des lors bien valoir sa braguette/ \& la feist au dessus esmoucheter de broderie a la Tudesque. Et le monde le louoit publicquement/ et en fut faict une chanson/ dont les petitz enfans alloient a la moustarde : \& estoit bien venu en toutes compaignies de dames \& damoyselles/ en sorte qu'il devint glorieux/ si bien qu'il entreprint de venir au dessus d'une des grandes dames de la ville/ (Pantagruel, 1532, XIV, p.115-116).

Tout ainsi qu'il se levoit pour ce faire apperceut a l'oree du boys ung beau grand chevreul/ qui estoit yssu du fort voyant le feu de Panurge/ a mon advis. Et incontinent se mist apres a courir de telle roiddeur/ qu'il sembloit que feust ung carreau d'arbaleste/ \& l'atrapa en moins d'ung riens/ et en courant tua des pieds dix ou douze que levraulx que lapins qui ja estoient hors de page. Doncq il frappa le chevreul de son malcus a travers la teste \& le tua/ \& en l'apportant recueillit ses levraulx. Et de tant loing que peust estre ouy/ il s'escrya/ disant. Panurge mon amy/ vinaigre vinaigre. (Pantagruel, 1532, XVI, p.135)

on en trouve ainsi 10 occurrences pour 17 points de jonction énonciative, le marqueur pragmatique prenant donc en charge près de $60 \%$ des articulations du discours. Pour autant, cette très forte densité constitue-t-elle un « événement de style »?

\section{Style d'auteur : « intensité » vs « décondensation »}

Du strict point de vue de la réception d'archive, l'histoire éditoriale du Pantagruel montre que les et de relance ont été perçus par les contemporains de Rabelais non comme une marque d'auteur, sanctuarisée et reconduite d'éditions en éditions, mais tout au contraire - comme une scorie intempestive. L'analyse des révisions du Pantagruel $^{39}$, de l'édition Claude Nourry de 1532 à celle de François Juste de 1542 en passant par l'édition (du même) de 1534, fait apparaître quelques données significatives : 140 occurrences de et en emploi de relance y ont été supprimées, suivant deux vagues successives - l'une, drastique, en 1534 (qui représente plus de $80 \%$ du total des suppressions), l'autre plus modérée, en $1542^{40}$. Aucun ajout - ou presque - ne vient par ailleurs pondérer ce processus d'épuration. Si, dans le Pantagruel, l'élimination des et de relance s'inscrit dans le vaste mouvement de réduction généralisée du nombre des marqueurs de structuration du discours (touchant également des connecteurs comme toutefois, car, ou mais), le petit mot de liaison à l'étude est affecté dans des proportions beaucoup plus importantes que les autres items. Cette logique se poursuivra, mais avec une intensité moindre, dans les révisions du Gargantua (qui comprend au demeurant, dès son édition princeps, des organisateurs textuels plus diversifiés que le Pantagruel ${ }^{41}$ ). Somme toute, l'histoire éditoriale du premier ouvrage de Rabelais se caractérise par un processus de décondensation, contraire au phénomène $d^{\prime}$ ' intensification » constitutif de «l'événement de style ${ }^{42}$ ».

\section{Langue littéraire et langue commune : de la « rupture » à la « continuité »}

Pour autant, du point de vue de leur production, les emplois de et de relance dans le Pantagruel n'en sont pas moins grammaticalement réguliers, et conformes aux usages 
contemporains. Dans son histoire de la coordination en français, Antoine (1962: 707-944, 707 et 920 notamment) a bien montré que le tour qui les mobilise, hérité du latin vulgaire, fréquent au Moyen Âge, est encore vivace sous la plume de tous les scripteurs renaissants, et dans tous types d'écrits (littéraires et non littéraires), du moins pour ce qui est de la coordination de phrases - la configuration $a, b, c$, et $n$, typiquement française, finissant par triompher en moyen français, pour ce qui concerne la coordination de syntagmes et de propositions. Le fait même que les grammairiens et remarqueurs du XVII e siècle prennent la peine de le défendre (voir notamment Vaugelas, ci-dessus, 2.1) prouve qu'il a encore une certaine productivité à l'aube du français classique. En usant du et de relance, la langue littéraire de Rabelais inscrit donc sa matière linguistique dans le prolongement de celle de la langue commune. La relation de celle-là à celle-ci n'est pas conflictuelle. Elle n'engage pas de changement de plan (ou de passage du palier sémantique au palier sémiotique), ne fait l'objet d'aucune «rupture». La langue littéraire se contente d'exploiter en les maximalisant toutes les potentialités de la langue commune. Loin d'être «l'autre » de cette dernière, elle en constitue, tout à la fois, l'illustration exemplaire et le parangon. Cette dialectique, qui s'exerce à l'intérieur d'un plan homogène, celui du niveau sémantique, sans solution de continuité, rapporte du même coup la littérature de la première modernité, caractérisée par ses très nombreux et de relance, à de l'écrit seulement parlé, et non vocalisé (ie, sémiotisé : voir ci-dessus, 2.1).

De ce point de vue, le corpus des «belles-lettres » ne se prête guère aux « événements de style ». Sa spécificité se laisse plus aisément saisir par les approches variationnistes de la littérarité.

\section{L'approche variationniste}

Si, dans l'écrit littéraire de l'Early Modern, les et de relance, faisant pourtant partie du canon linguistique de l'époque ${ }^{43}$, n'en constituent pas moins un phénomène saillant, sans doute est-ce en vertu du jeu variationnel auquel ils soumettent leur(s) contexte(s) d'accueil. Nous en donnerons trois illustrations, à chaque fois un peu différente.

\section{Rabelais et la variation textuelle}

Dans l'édition princeps (1532) du Pantagruel, le recours aux et de relance, particulièrement fréquent, n'est pas uniformément réparti : contrairement à toute attente, le marqueur d'oralité n'apparaît guère dans les passages de discours rapporté (voir Badiou-Monferran \& Monferran, à paraître). Il figure, principalement, dans les séquences narratives, textuelles (voir ci-dessus, ex. 25) ou paratextuelles (telles les titres de chapitre ${ }^{44}$ ou encore le Prologue) :

(26) Mais que diray je des pauvres verollez \& goutteux? O quantesfois nous les avons veu a l'heure qu'ilz estoient bien oingtz \& engressez à point/ \& le visaige leur reluysoit comme la claveure d'ung charnier/ \& les dentz leurs tressailloient comme font les marchettes d'ung clavier d'orgues ou d'espinette quand on joue dessus/ \& que le gousier leur escumoit comme a ung verrat que les vaultrez et levriers ont chasse sept heures : que faisoient ilz alors? toute leur consolation n'estoit que de ouyr lire quelque page dudict livre. Et en avons veu qui se donnoient a cent pippes de diables/ en cas qu'ilz n'eussent senty allegement manifeste a la lecture dudict livre [...]. (Rabelais, Pantagruel, 1532, Prologue : n.p.) 
Pour autant, on ne saurait en faire un trait idiolectal d'Alcofribas, le narrateur. De fait, les rares passages de discours direct où ce dernier prend la parole en tant que personnage de son propre récit - à savoir, lors de sa visite des églises parisiennes avec Panurge (chap.XII) et lors de son expédition à l'intérieur du corps du géant (chap. XXII) - ne comprennent pas de et de relance ${ }^{45}$. L'occurrence du MP est donc associée, dans le Pantagruel, à la fonction narratoriale. Cette restriction d'emploi, qui constitue une variation par rapport à l'usage commun contemporain, sollicitant le marqueur d'oralité dans tous types de configuration (énonciation historique, énonciation de discours), mais tout particulièrement dans le discours rapporté, confère au petit mot de liaison une saillance bien particulière. Attirant de ce fait l'attention des lecteurs, ce dernier apparente la stratégie communicationnelle du narrateur rabelaisien à celle d'un bonimenteur. En tant que régulateur phatique, inscrivant les vertus du discours oral (notamment son "émotionnalité forte", son "ancrage actionnel et situationnel ", sa "spontanéité », sa "liberté thématique», sa " coopération communicative intense ${ }^{46}$ ») au cœur de l'acte de narration, il transforme le récit écrit en performance. La littérarisation / stylisation du Pantagruel s'effectue ainsi sans solution de continuité, à l'intérieur du seul plan sémantique, par un jeu de variation proportionnelle entre l'occurrence d'un fait de détermination langagière (celui du et de relance) et le type de texte (le récit - et non le discours) qui lui est, contre toute attente, associé.

\section{Molière et la variation formelle}

L'incipit du Dom Juan (représenté en 1665 mais publié pour la première fois en 1682 dans l'édition Denis Thierry, Claude Barbin et Pierre Trabouillet ${ }^{47}$, que nous reproduisons ci-dessous ${ }^{48}$ ) ménage un autre type de surprise. S'il contient dès sa première phrase-période une occurrence de et de relance après un signe de ponctuation fort :

(27) SGANARELLE, tenant une Tabatiere. Quoy que puisse dire Aristote, et toute la Philosophie, il n'est rien d'égale au Tabac, c'est la passion des honnestes gens; et qui vit sans tabac n'est pas digne de vivre; non seulement il réjouït et purge les cerveaux humains; mais encore il instruit les ames à la vertu [...] (Molière, Dom Juan, 1682, I, 1)

l'apparition du MP, marqueur d'oralité, ne constitue ici en rien une curiosité linguistique, puisque le texte dramatique, consignant des propos proférés, a par définition partie liée avec l'oral représenté49. Plus étonnant en revanche est sa combinaison, dans le cadre d'une pièce en prose, avec un vers blanc, qu'il lance (« et qui vit sans tabac // n'est pas digne de vivre »). Sans doute s'agit-il d'un alexandrin : vers réputé prosaïque et qui, ainsi que l'a montré Fournier (2009) à propos de la récriture versifiée du Dom Juan de Molière par Thomas Corneille, se prête à une structuration "bi-modulaire», opposant, à l'image des énoncés de l'oral spontané d'hier et d'aujourd'hui, un "préambule» long et complexe - constitué d'un ligateur (et), d'un " thème » (qui vit) et d'un "cadre " (sans tabac) - à son élément rhématique (n'est pas digne de vivre $)^{50}$. Toutefois, le segment textuel de 12 syllabes fait ici l'objet d'une organisation métrique renforcée, qui le distingue des énoncés oraux ordinaires et le rend à sa nature de vers. Cette dernière est en effet exhibée (a) par une structuration syntaxique apparentant l'énoncé à un tétramètre (« et qui vit [3 syllabes]/ sans tabac [3 syllabes]// n'est pas di/gne de vivre [rhème : 2 fois 3 syllabes]); (b) par le polyptote « vit/ vivre ", dont les deux termes, placés respectivement en début et en fin d'énoncé, 
assurent l'ouverture et la clôture ; (c) par la progression sonore en chiasme [i] [a] [a] [i] (qui vit /tabac; n'est pas / digne ; vivre) qui en scelle l'unité et la cohésion. Et, élément optionnel, ici mobilisé pour parvenir au compte des 2 fois 6 , se signale donc, en l'occurrence comme une ligature assurant la jonction de la prose et du vers. Il constitue, à ce titre, un introducteur de variation formelle, ouvrant la voie à la récriture versifiée de la pièce par Thomas Corneille ${ }^{51}$. Dans cette dernière, on retrouve le vers blanc de Molière, mais à une place terminale, qui convertit la relance en clôture, et le MP en connecteur conclusif ${ }^{52}$ :

(28) Quoy qu'en dise Aristote, et sa digne Cabale, Le Tabac est divin, il n'est rien qui l'égale,

Et par les Fainéants pour fuir l'oisiveté

Jamais amusement ne fut mieux inventé.

$[\ldots]$

Mais c'est peu qu'à donner, instruisant la Jeunesse,

Le Tabac l'accoûtume à faire ainsi largesse.

C'est dans la Medecine un remede nouveau ;

Il purge, réjoüit, conforte le cerveau,

De toute noire humeur promptement le délivre,

Et qui vit sans Tabac n'est pas digne de vivre.

O Tabac, ô Tabac, les plus cheres amours!

Mais reprenons un peu nostre premier discours. (Th. Corneille, Le Festin de Pierre,

$1683, \mathrm{I}, 1)$

À l'intérieur du niveau sémantique, et sans solution de continuité avec les usages de la langue commune, le et donjuanesque se fait donc le support d'une double variation: une variation formelle, intratextuelle et horizontale chez Molière, en tant que tremplin de la prose au vers; une variation catégorielle, intertextuelle et verticale chez Corneille, substituant à l'emploi originel de MP un emploi de connecteur. Cette richesse variationnelle, qui, opérant à l'intérieur du seul niveau sémantique, ne se laisse guère saisir par la grille de "l'événement de style», est ce qui contribue pour partie à l'artistisation (la littérarisation) des productions lettrées qui le mobilisent. Les contes de Perrault reconduisent le type de variation dont joue ici Thomas Corneille, mais en en projetant les réalisations et les effets sur l'axe, horizontal, des enchaînements propositionnels qui composent le texte.

\section{Perrault et la variation catégorielle}

Dans l'extrait ci-dessous, emprunté à La Belle au bois dormant, la mère du Prince charmant, issu d'une famille d'ogres, s'apprête à dévorer la première de ses petits enfants :

(29) [Elle] dit un soir à son Maistre d'Hôtel, je veux manger demain à mon dîner la petite Aurore. Ah! Madame, dit le Maistre d'Hôtel ; je le veux, dit la Reine ( $\& 53$ elle le dit d'un ton d'Ogresse, qui a envie de manger de la chair fraische), $\&^{54}$ je la veux manger à la Sausse robert. Ce pauvre homme voyant bien qu'il ne falloit pas se joüer à une Ogresse, prit son grand cousteau, $\&^{55}$ monta à la chambre de la petite Aurore : elle avoit pour lors quatre ans, $\$^{56}$ vint en sautant \& en riant se jetter à son col, \& luy demander du bon du bon. Il se mit à pleurer, le couteau luy tomba des mains, $\& 57$ il alla dans la basse-cour couper la gorge à un petit agneau, $\&^{58}$ luy fit une si bonne sausse que sa maistresse l'assura qu'elle n'avoit jamais rien mangé de si bon. (Perrault, Histoires ou Contes du temps passé, « La Belle au bois dormant. Conte », 1697, Paris, Barbin : 35) 
Sous couvert d'une linéarisation identique associant par \& deux propositions ou massifs de propositions, Perrault, jouant de la polyfonctionnalité de et, expérimente avec virtuosité tous les types d'enchaînements que cet item, sémantiquement sousdéterminé et de ce fait plus aisément transcatégorisable ${ }^{59}$, est susceptible de spécifier :

(i) enchaînement à l'intérieur du discours direct où et, fonctionnant comme un MP, est optionnel, et vient servir un effet de relance (hiérarchisant, dans le cadre d'une énonciation à double détente, l'énoncé commenté et son commentaire - voir 29.1 -, ou l'énoncé de base et sa récriture développée - voir 29.2).

(ii) enchaînement à l'intérieur de la séquence narrative où et, assurant par transcatégorisation une fonction de connecteur, est plus difficilement supprimable, et marque une relation implicative de nature tantôt temporelle (voir 29.3), tantôt consécutive (voir 29.4 et 5), tantôt conclusive (voir 29.6), au sein d'un bloc sémantique énonciativement homogène, non récursif, et en ce sens, clos sur lui-même.

(iii) A quoi s'ajoutent les enchaînements syntagmatiques au sein desquels et, fonctionnant, en vertu de sa valeur de base, non pas comme un MP ou un connecteur, mais comme un coordonnant ${ }^{60}$, associe, contrairement aux autres cas, des conjoints potentiellement réversibles ( « en sautant et en riant » > " en riant et en sautant »; " se jeter à son col et lui demander du bon du bon » > «lui demander du bon du bon et se jeter à son col »).

Le processus de littérarisation/stylisation du conte tient ici à la sur-sollicitation d'une même construction en $\mathrm{X}$ [proposition ou syntagme] et $\mathrm{Y}$ [proposition ou syntagme], dans des contextes syntaxiques, sémantiques et énonciatifs qui, sans s'écarter des formats mobilisés par ailleurs dans l'écrit ordinaire, introduisent de la variation en explorant toutes les portées (syntagmatiques vs propositionnelles) et toutes les valeurs (coordonnantes, connectives, discursives) du petit mot de liaison disponibles en langue, à l'intérieur d'un espace textuel pourtant limité à trois phrases-périodes. En cette fin de XVIIe siècle où le MP est désormais presque toujours cantonné aux seuls segments d'oral représenté (tels le discours rapporté direct et l'incise de commentaire dans l'extrait ci-dessus), l'emploi de relance ne devient coruscant que par différence, dans le jeu de l'étourdissante trans-catégorisation qui le lie aux différents emplois de clôture (emplois de connecteur conclusif, consécutif, temporel) de son environnement immédiat.

Somme toute, dans le cadre de l'ancien régime littéraire, où les belles-lettres relèvent encore de l'écrit parlé, et non vocalisé, ie, d'un écrit modelé sur l'oral, la saillance stylistique d'un marqueur d'oralité comme le et (de relance) ne fait pas en soi "événement ». L'occurrence du trait de détermination langagière en question, qui s'exerce à l'intérieur du seul niveau sémantique, demeure indifférente aux processus de sémiotisation et sur-sémiotisation. Sa puissance esthétique est proportionnelle aux effets de variation dont et fait l'objet, introduisant de la surprise et déjouant les attentes. Aussi l'Early Modern oppose-t-il :

(i) à la propriété de rupture constitutive de l'événement de style, celle de la proportionnalité : à l'intérieur d'un plan homogène (en l'occurrence, le plan sémantique), l'effet (le degré de stylisation) semble bien devoir être proportionnel à la cause (le degré de variation sollicité).

(ii) à la propriété d'intensification celle d'additivité, supposant que c'est la mobilisation conjointe de l'échantil et de ses variantes qui créera de la valeur, le tout étant égal à la somme des parties. L'avènement stylistique (plus que l'événement de style) se définira, pour 
lors, en extension, par la totalisation des faits de variation et co-variation qu'il comporte $^{61}$.

Sans vouloir à tout prix réconcilier l'ancien et le nouveau régime stylistique, ou, au plan épistémologique, l'approche variationniste du style, très efficace pour le corpus des belles-lettres, et l'approche événementielle, opératoire pour la littérature moderne, on se demandera sous quelles conditions une histoire continue du changement stylistique, effectuée en diachronie longue, sur un empan conduisant du XVIe au XXIe siècle, est envisageable. Nous formulerons l'hypothèse que le cadre émergentiste, susceptible d'accueillir et articuler en son sein logique événementielle et logique variationniste, constitue une réponse possible.

\section{Histoire du changement stylistique et approche émergentiste du style}

Le concept d'émergence, qui advient comme tel pour la première fois sous la plume de Lewes (1875), ne s'élabore véritablement qu'au premier tiers du $\mathrm{XX}^{\mathrm{e}}$ siècle, sous l'impulsion de Bergson (1907) et de philosophes britanniques (Alexander 1920 ; Morgan 1923 ; Broad 1925 ; Whitehead 1929). De l'étymon latin emergere, il retient deux traits, celui de manifestation et celui de nouveauté, et constitue ainsi le cœur d'une « doctrine de l'avènement de la nouveauté » (Morgan, cité dans Fagot-Largeault 2002 : 984-985). Partant du constat qu'il existe, dans la nature, des « effets qui ne se réduisent pas à l'addition mécanique de leurs causes " - tels la vie, le psychisme, la pensée réflexive (Fagot-Largeault, 2002: 984-985), il définit ces effets comme des éléments " émergents » et les oppose aux éléments "résultants " qui, pour leur part, sont calculables, du moins rétrospectivement, à partir de leurs causes. Il faudra attendre la fin du XXe siècle pour que le concept d'émergence accède au statut de "nouveau paradigme ", c'est-à-dire de nouveau système de pensée, dans des domaines aussi différents que la biologie, la médecine, la psychologie des comportements ${ }^{62}$, ou encore la physique ${ }^{63}$. Les linguistes ont pour leur part commencé à s'intéresser à la théorie de l'émergence à partir de la fin des années $1980^{64}$.

\section{Le paradigme émergentiste : quelques rappels ${ }^{65}$}

En tant que paradigme, l'émergentisme s'oppose aux théories réductionnistes, ou déterministes, qui considèrent que, dans un état de choses donné, toute nouveauté est rapportable à un régime de détermination explicitement identifiable à partir d'éléments observables dans l'état de choses antérieur. Alors que le déterminisme repose sur le postulat d'une continuité causale reliant l'état de choses initial et l'état de choses immédiatement subséquent, à l'inverse, l'hypothèse émergentiste formule le postulat d'une discontinuité entre celui-ci et celui-là. Le paradigme émergentiste constitue ainsi un «modèle dynamique non linéaire » (Ripoll 2005 : 213), ou encore, une synthèse imprévisible, qui suppose :

(i) la non additivité des causes. Le tout n'est pas égal à la somme des parties, les effets s'expliquent par les causes, en dépendent, mais ne s'en déduisent pas pour autant, en vertu des interactions desdites causes les unes sur les autres, qui compliquent et obèrent le mécanisme de déduction.

(ii) l'hétérogénéité des effets et des causes. Celles-ci et ceux-là appartiennent à des 
ordres, ou à des plans différents. Les propriétés séparées de l'oxygène et de l'hydrogène ne renseignent pas correctement sur les propriétés de l'eau ;

(iii) l'accroissement de complexité. Synthétiques, les effets appartiennent à un palier de niveau supérieur à celui des causes. Il y a eu «progrès de l'organisation " (loc. cit. par Fagot-Largeault $2002: 961)^{66}$.

(iv) l'influence potentielle des effets sur les causes. Les phénomènes du niveau inférieur peuvent être influencés par ce qui se passe au niveau global (effets de contexte) ${ }^{67}$.

On est alors en droit de se demander quelle mesure cette nouvelle conceptualisation de l'innovation permet de prendre en charge, tout à la fois, l'approche variationniste et l'approche événementielle du style, et de rendre compte de leurs lignes de passage, dans le cadre d'une réflexion sur l'histoire du changement stylistique.

\section{Émergence et événement de style}

L'émergentisme croise sans surprise la problématique de «l'événement de style ».

\section{"Non additivité des causes » vs « imprévisibilité »}

En tant que modèle explicatif antiréductionniste, postulant la non additivité des causes, il entre à l'évidence en intersection avec le premier critère définitoire de l'événement de style, celui de "l'imprévisibilité », défini comme suit par les co-éditeurs du présent numéro: «L'événement s'annonce dans une absolue nouveauté, dans la mesure où il semble inexplicable à partir des possibles qui lui préexistent » (Watine \& Yocaris 2016).

À cet égard, dans le vaste champ des pratiques imitatives ${ }^{6}$, le pastiche constitue une expérience limite, permettant de tester la validité de cette hypothèse. La combinaison d'un rythme anapestique, du temps verbal de l'imparfait et d'un et de relance, source de la vocalité flaubertienne (voir ci-dessus, 2.2.1), donnera lieu à un pastiche de Flaubert, non à du Flaubert - aussi talentueux que soient ses imitateurs (voir ici même sous 2.2.1, la prose de Cocteau et de Perec $\left.{ }^{69}\right)$. Autrement dit, le «style » de Flaubert n'est pas équivalent à l'imitation de sa signature. Du point de vue de la tradition (allemande) de la «stylistique des styles ${ }^{70}$ » en effet, tradition qui apparente le concept de «style » à un projet communicationnel, celle-là (la marque de fabrique) ne saurait par définition contenir celle-ci (la vision du monde): sauf cas particulier ${ }^{71}$, l'idiolecte, a priori « involontaire » (Philippe 2005 : 82) et du coup dépourvu de « contenu de signification » (ibid.) ne participe pas, même localement, à la construction de l'interprétation. Il se situe donc en déport de l'objet "style", pour sa part associé à une stratégie informationnelle, qu'il vient servir. Le pastiche, qui mobilise l'idiolecte, passe à côté du style d'auteur, «imprévisible ». De même, du point de vue (cette fois franco-français ${ }^{72}$ ) de la "linguistique des styles", identifiant à l'inverse, pour tout ou partie, style d'auteur et marque de fabrique (voir Philippe 2005 : 84), l'imitation de l'idiolecte n'en est pas moins distincte de l'idiolecte. Comme l'ont souligné - après Genette (1992) Petitjean (1984), Philippe (2005) et Denis (2012), le pastiche se signale par sa "saturation" (Denis 2012:9) - ou encore, sa "densité " (Philippe 2005: 88) exceptionnelle. Pour être perçus comme tels, les traits de détermination langagiers imités sont sur-sollicités, ie, intensifiés et condensés à l'intérieur d'un espace textuel tout entier dévoué à la réalisation de son projet imitatif. Gilles Philippe commente de facto le pastiche des Goncourt par Proust en ces termes: 
«[...] aucune page du journal des Goncourt ne ressemble au pastiche qu'en donne

Proust » (Philippe $2005: 88$ ),

tant le « poids » (ibid.) des traits imités en est lourd et la "portée » (ibid.) suffisamment longue pour que la marque idiolectale ne soit pas diluée «au saut de phrase ou de paragraphe ", et, à l'inverse, "ne soit pas atténué[e] ou annulé[e] par la récurrence " (ibid.). Moins serré, plus relâché, le vrai style d'auteur ne se laisse pas saisir par le repérage et la réduplication de ses traits idiolectaux dans le cadre du pastiche. Il constitue bien, à ce titre, une synthèse "imprévisible", non reproductible, même rétrospectivement à partir du repérage des éléments qui le constituent. Avérée pour toutes les époques (la Modernité mais aussi l'Early Modern ${ }^{73}$ et la postmodernité), cette propriété essentielle et transhistorique de l'objet «style " semble aussi bien pouvoir être ressaisie par la grille événementielle (qui l'appréhende en termes d'imprévisibilité) que par la grille émergentiste (qui propose de la décrire comme un cas de noncompositionnalité).

\section{" Hétérogénéité des effets et des causes » vs « rupture »}

Si le modèle émergentiste ne théorise pas la question de "l'intensité ", pourtant centrale dans l'appréhension des phénomènes d'artistisation ${ }^{74}$, et s'il se situe du coup un peu en retrait de la proposition de "l'événement de style ", qui, pour sa part, la prend en charge (voir ci-dessus, 2.2.), il confère en revanche au critère de la « rupture », défini en ces termes par les coordinateurs du présent numéro :

« Rupture : l'événement représente un tournant radical dans l'expérience : “après le

surgissement de l'événement, ce ne sera plus jamais comme avant (Romano 1998 :

62)" " (Watine \& Yocaris 2016),

un cadre d'analyse fécond. Le principe de l'hétérogénéité des effets et des causes présente de facto l'avantage :

(i) d'expliciter la nature de la "rupture " à l'œuvre dans l'événement de style, en faisant valoir le saut qualitatif (du sémantique au sémiotique) qui le caractérise. Ou, pour le dire autrement, en rendant compte du fait que les propriétés des observables parmi d'autres, le MP et, le temps de l'imparfait, ou encore, le rythme ternaire - ne renseignent pas correctement sur celles du produit émergent-en l'occurrence, la prose subjectiviste des années 1850 (voir ci-dessus 2.1).

(ii) de localiser la cible de la rupture (l'émetteur, le récepteur ou le produit émergent) : c'est bien ici le texte-discours (certes émis et reçu) qui, dans ce cadre, se trouve affecté par l'événement. L'« émergence » stylistique semble avant tout ontologique. L'enjeu de sa modélisation réside principalement dans l'identification - et la caractérisation - de "spécificités textuelles objectivables ${ }^{75}$ »: en l'occurrence, dans l'exemple qui nous occupe ici, en la caractérisation des faisceaux de déterminations langagières accompagnant les différentes manifestations du paradigme vocalisant.

La reprise phénoménologique ${ }^{76} \mathrm{du}$ processus émergent, sollicitant le pôle de la réception (et de la réception impliquée notamment) n'est bien entendu jamais impossible ${ }^{77}$. Dans certaines configurations, en appelant à une version continuiste de l'émergence (voir ci-après, 4.3), elle constitue même une condition nécessaire à l'avènement d'un produit émergent. C'est ce cas de figure qu'illustrent les productions lettrées de la première modernité, soumises entre autres à l'idéal (anti-événementiel et, ontologiquement, anti-émergent) de la variation. 


\section{Émergence et approche variationniste du style}

Dans quelle mesure le modèle émergentiste est-il susceptible de rendre compte du «style» des productions lettrées de la première modernité ? Qu'apporte-t-il - et qu'apporte-t-il de plus que la proposition événementielle - à l'approche variationniste, bien adaptée à ce corpus de texte? On rappellera ici que la théorie émergentiste suppose à la fois "une continuité et une discontinuité, continuité réelle des composants [ie, des observables], discontinuité apparente [des observés] (BadiouMonferran \& Ducos 2012: 3). Si l'on privilégie, comme on vient de le faire, la composante "discontinue », en sélectionnant les deux propriétés de l'imprévisibilité (ou «non-additivité des causes») et du saut (ou "hétérogénéité des effets et des causes »), le modèle risque fort d'être inadéquat. $\mathrm{Si}$, en revanche, à la suite d'un certain nombre de linguistes (voir notamment Soutet, Nyckees et Rastier dans BadiouMonferran \& Ducos 2012), on inverse la perspective et qu'on privilégie le «continuum entre l'immergé et l'émerge(a)nt/émergé » (Soutet 2012 : 39), en se focalisant sur les deux dernières propriétés de l'«accroissement de complexité » et de l'«influence potentielle des effets sur les causes" (voir 4.2 (iii) et (iv) ci-dessus), la reprise émergentiste de l'approche variationniste redevient possible.

\section{Variation et « accroissement de complexité »}

La valeur ajoutée du critère de l'« accroissement de complexité », constitutif de modèle de l'émergence, à l'approche variationniste du style, est selon nous que ce critère permet de saisir, en diachronie, à la faveur d'un retournement privilégiant le pôle de la réception sur celui de la production, un phénomène stylistique souvent éprouvé mais imparfaitement documenté : celui du feuilleté interprétatif, surimposant au contexte de production initial, que la réception d'archive se donne pour objet de reconstituer ${ }^{78}$, la somme des réceptions impliquées ${ }^{79}$ - potentielles et/ou effectives - qui sont venues progressivement enrichir ce dernier. La critique désigne généralement ce processus sous le nom d' «actualisation ${ }^{80}$ " - et la proposition événementielle (mais dans une perspective a priori plus synchronique que diachronique) sous celui «d'adresse ${ }^{81}$ ». L'apport, selon nous, de la glose émergentiste est qu'elle met en évidence les diverses propriétés de cette réception en feuilleté : uni-directionnalité, conduisant du simple au compliqué ; reconfiguration, sur le long cours, de la prévisibilité variationniste en synthèse imprévisible; diffraction, en diachronie toujours, de l'homogénéité variationniste de départ en une organisation multi-strates, comprenant des propriétés d'échelles différentes, suivant un modèle fractal. La fortune de l'incipit du Dom Juan de Molière au sein de l'histoire littéraire française viendra ici servir d'observatoire.

En réception d'archive, l'éloge paradoxal du tabac, qui ouvre la pièce, et qui s'articule autour d'un et de relance conjoignant prose et vers blanc (voir ci-dessus), a pu être lu comme " un hors-d'œuvre d'actualité », contenant " une satire contre les dévôts " ${ }^{82}$. Introduit en Europe par les espagnols en 1560, le tabac est de facto interdit à la vente en France à partir de 1635. Le règlement de police de Paris n'en autorise plus l'achat que chez les apothicaires, afin des fins thérapeutiques, contre ordonnance médicale. Le Pape Urbain VIII (mort en 1644) menace au même moment d'excommunication les fumeurs, tout particulièrement ceux qui prisent dans les églises. Pour sa part, la compagnie du Saint-Sacrement (autrement dit, le "parti dévot») condamne l'usage d'une substance incompatible avec le christianisme, « car le lien est trop étroit entre le 
tabagisme, l'idolâtrie et les pratiques inquiétantes des chamanes amérindiens... Ce qui émane de barbares ne peut être que barbare : fumer, croit-on, est un artifice du Diable pour amadouer et tromper les chrétiens (Von Gernet, 1995)» (Ferland 2007 : 35). C'est ce contexte que relayera la récriture en vers de Dom Juan par Thomas Corneille. La conclusion introduite par et (connecteur - voir ci-dessus 3.2.2) « Et qui vit sans tabac n'est pas digne de vivre " s'inscrit dans la subséquence d'un argumentaire ne justifiant la consommation du tabac que comme médicament («C'est dans la Medecine une remede nouveau »). Elle doit être lue au sens propre, comme apologie d'un traitement médical augmentant l'espérance et la qualité de vie des priseurs. Par ricochet, dans ce contexte, en réception d'archive, on sera tenté de réduire la « relance » initiée par et dans le texte original de Molière à un jeu formel - où le MP constituerait un simple tremplin autorisant passage de la prose au vers (voir ci-dessus 3.2.2). La redécouverte du texte de Molière au XIX ${ }^{\mathrm{e}}$ siècle, occulté pendant près de 150 ans par la récriture versifiée de Thomas Corneille, va rouvrir le débat sur le sens de son étrange incipit. A la réception d'archive, s'adjoint désormais toute une série de lectures nouvelles. Parmi elles, se détachent (i) l'interprétation structurale de Serres (1968) qui voit dans l'éloge paradoxal du tabac un "modèle réduit» (ibid. : 234) de la pièce, contenant «tout, le canevas, la règle, la menace, la fin [...]. Les trois conduites de Dom Juan, vis-à-vis des femmes, du discours, de l'argent, forment trois variations parallèles sur le thème du tabac» (ibid.: 235) (ii) une interprétation de type métadiscursif, qui, dans l'éloge paradoxal du tabac, voit un éloge doxal du théâtre. La référence à Aristote, la proximité sonore des mots "tabac"/théâtre ", l'idée de "purgation" des "passions" et "d'instruction [des âmes] à la vertu ", rappelant la catharsis, vont de facto dans ce sens. De ce feuilleté interprétatif, il résulte un accroissement de complexité, qui se caractérise :

(a) par une dynamique unidirectionnelle, conduisant du simple au compliqué : l'incipit dit désormais plus que le message satirique (prenant le contre-pied des positions du parti dévot) programmé par son contexte de production.

(b) par son caractère imprévisible : l'interprétation structurale et l'interprétation métaénonciative n'étaient pas contenues en germe dans la réception princeps. La récriture de Corneille, qui suspend la possibilité de l'une et de l'autre en verrouillant la lecture satirique comme seule lecture possible, le montre.

(c) par la diffraction, suivant un principe fractal, du plan homogène (de nature sémantique) articulant, en réception d'archive, les effets et les causes (voir ci-dessus 3.2.2). De l'édition princeps aux éditions modernes, autrement dit, de l'échelle synchronique à l'échelle diachronique, la lettre du texte de Molière demeure, à peu de choses près, inchangée - exception faite des variantes ortho-typographiques et, dans une moindre mesure, lexicales (sur cette question, voir le site "Molière 21 " qui compare les deux premières éditions du Dom Juan - celle de 1682 et celle de 1683). Cette stabilité est ce que le modèle fractal nomme l'invariant d'échelle. Pour autant, le passage du point de vue synchronique à un point de vue diachronique, de la réception d'archive à une réception impliquée, déclenche des propriétés d'échelle différentes. Le régime impliqué sémiotise ainsi la lettre du texte. Soit qu'il donne à lire l'éloge paradoxal du tabac comme un signal transgressif, programmant toutes les transgressions donjuanesques de la pièce. Soit qu'il reconfigure l'incipit en commentaire "suiréflexif » (Benveniste 1966: 274), mobilisant le principe de la double énonciation pour faire l'éloge du théâtre sous celui du tabac. Dans tous les cas, le changement d'échelle (avec le passage d'une visée synchronique à une visée diachronique, tenant compte de 
toutes les lectures ayant assuré et continuant d'assurer la transmission de la pièce) introduit du jeu dans le plan sémantique de la lettre du texte. Les effets et les causes ne relèvent plus du même niveau.

$\mathrm{Si}$, du point de vue de la production, les nombreux effets de style de Dom Juan, associés aux jeux variationnels opérant au niveau sémantique, et calculables à partir d'eux, ne relèvent pas de la catégorie de "l'événement de style », du point de vue leur réception en feuilleté, le critère de "l'accroissement de complexité ", mobilisé dans le modèle émergentiste (mais pas dans les autres modélisations) présente le mérite de pouvoir prendre en charge et de décrire leur « événementialisation ».

\section{Variation et influence potentielle des effets sur les causes}

La reprise de l'incipit du Dom Juan de Molière dans le cadre de sa réception en feuilleté (l'effet) n'est pas sans incidence sur la matière textuelle qui en constitue la source (ou la cause). Dans le cadre des lectures impliquées évoquées plus haut, le MP et de « et qui vit sans tabac n'est pas digne de vivre » devient plus qu'un tremplin variationnel, faisant communiquer prose et vers. Il indexe, en tant qu'élément de relance, toutes les relances interprétatives (de type structural et/ou méta-discursif) auxquelles se prête précisément l'incipit. Bref, il acquiert un statut de symbole (au sens de Peirce).

\section{Conclusion}

Élaborée en partie pour l'analyse des fictions postmodernes, la proposition de "l'événement de style " s'avère, dans ce cadre, provocatrice et opératoire (ou opératoire car provocatrice). Elle prend en effet à rebours la position dominante selon laquelle les fictions littéraires contemporaines d'expression française, visant l'horizon d'un « redevenir-discours » (Piat, dans Philippe \& Piat 2018), font moins « événement » par leur style que par leur contenu référentiel : «Le scandale ne vient pas (ne vient plus) d'un auteur qui écrit mal (ou, plus globalement, de la façon dont un auteur écrit), il vient de ce dont traitent ses œuvres (voir Soumission de Houellebecq); il vient d'une posture médiatique [...] d'un positionnement idéologique » (Piat, ibid.)

Dans ce cadre - qui rompt délibérément avec l'entreprise d'autonomisation de la langue littéraire à laquelle il succède - l'idéal d'une "écriture courante " (selon les mots par lesquels Duras qualifie sa prose des années 1980), rencontrant «la langue orale la plus communément partagée » (Piat, ibid.), semble s'être partout (et chez tous) imposé. Pour autant, « la littérature d'aujourd'hui n'est pas plus naïve que celle d'hier : d'une part, les héritages peuvent se dire ou se montrer, avec une tendance à retrouver sous la plume d'un auteur celle d'un autre (Beckett chez Chevillard; Claude Simon chez Éric Laurrent) comme si, malgré tout, l'imaginaire auteuriste du style était encore présent, comme rémanent [...] D'autre part, [et plus généralement...] la langue actuelle des écrivains [...] réemploie des configurations particulièrement visibles à un moment de l'histoire de la langue littéraire » (Piat, ibid.).

La proposition de "l'événement de style " constitue ainsi un garde-fou salvateur, permettant de dissocier l'« imaginaire de la langue commune » constituant l'horizon de la langue littéraire contemporaine, et la « langue commune » elle-même, autrement dit, le « patron » et l'« appareil formel ${ }^{83} »$. Un fragment comme : 
(30) La gaufrette s'était émiettée et il avait fini par la jeter. Avait grandi, était parti, était revenu et elle avait ri. Et il l'avait cru. Et il avait vieilli, et il avait grossi et...et elle était morte. Et voilà. (Gavalda, La Consolante, 2008 : 172)

rejoint sans doute les récits oraux de vie quotidienne - ou les écrits non littéraires - du fait de la présence des et de relance, des points des suspension matérialisant les blancs du discours, du jeu des répétitions (et...et), restituant le bégaiement d'une parole construisant sa progression dans la contemporanéité de son énonciation, de la mobilisation de particules de discours résomptives - «Et voilà»-, lexicalement pauvres mais pragmatiquement efficaces. Il s'en éloigne de la même manière à cause de sa densité informationnelle, qui l'apparente à la figure narratologique du " sommaire », de sa syntaxe parfois "a-subjective» («Avait grandi, était parti, était revenu») rompant avec la tradition orale d'expression du sujet, du recours au plus-que-parfait (en lieu et place du passé composé privilégié dans les récits parlés). L'« événement de style » tient ici à la sémiotisation de traits certes issus de la langue commune, mais que leur association avec des éléments étrangers à «l'écriture courante » - ou à l'oral invite à requalifier: faisant retour sur eux-mêmes, les ingrédients du "parler » se représentent, au second degré, comme symptôme du nouvel idéal linguistique qui caractérise le régime littéraire français contemporain : celui, "post-autonome », du « redevenir discours » de la littérature (Piat).

Si, dans la perspective d'une histoire du changement stylistique à l'envers, on interroge la pertinence de la proposition événementielle pour la période précédente, celle de la modernité littéraire conduisant de Duras à Flaubert, on pourra prendre toute la mesure de l'opérativité du modèle. C'est sans surprise qu'en ce moment d'autonomisation de la langue littéraire, l'hypothèse de l' « événement de style » fonctionne à plein régime, et est continument validée, sur le mode de la «suite ininterrompue d'événements (successifs et/ou simultanés) $»^{84}$ (voir ci-dessus, la partie 2).

Pour la période de l'Early Modern en revanche, qui, localement, n'est pas exempte d' "événements de style » (comme en témoignent, parmi d'autres, les prétentions de la Pléiade à l'invention d'un nouveau langage poétique, radicalement différent du précédent), mais qui, globalement, pense la langue littéraire comme une illustration accomplie de la langue commune, et le style, comme le support et la manifestation d'un idéal de variation, la proposition événementielle devient inopérante. Et c'est ici que, pour qu'on puisse se saisir de ce "reste », le recours à l'approche émergentiste peut s'avérer utile. Non qu'en réception d'archive il soit d'un quelconque secours. Tout comme l'événement de style, l'émergentisme échoue à décrire l'esthétique variationnelle propre à l'Early Modern. Mais à la faveur d'un changement d'échelle, mobilisant l'entier du feuilleté interprétatif grâce auquel les productions lettrées de la première modernité nous parlent encore aujourd'hui, il présente l'avantage de pouvoir décrire avec précision, d'un point de vue diachronique, les conditions de leur "événementialisation". Sans doute manque-t-il, en l'état, à l'hypothèse de "l'événement de style ", la dimension historique et historicisante qui lui permettrait de combler ce vide. Sur cette question, le modèle émergentiste apporte, quant à lui, des éléments de réponse, et c'est pourquoi nous plaidons pour la poursuite du dialogue entre l'une et l'autre proposition.

Nous reviendrons, pour finir, sur ce qui constitue le facteur d'intersection le plus important entre les deux modélisations : le critère de la "non-additivité des causes " dans un cas, glosé par celui de "l'imprévisibilité " dans l'autre. Aux questions du « pourquoi » et du « comment » cela évolue, du « pourquoi » et du « comment » cela se 
transmet, du «pourquoi » et du «comment» cela disparait, l'une et l'autre ne prétendent apporter aucune réponse définitive. La stylistique historique que ces deux propositions appellent de leur vœu est probabiliste. L'une de ses tâches à venir, pour ces prochaines décennies, est sans doute l'établissement, pour les événements de style, de scénarios d'apparition, disparition et survie. Cette entreprise " explicative ", au sens certes probabiliste du terme, semble d'autant plus nécessaire selon nous que les productions lettrées, d'hier et d'aujourd'hui, font de plus en plus l'objet de "trigger warnings ${ }^{85} »$. Autrement dit, d'avertissements - signalant dans les œuvres littéraires un contenu potentiellement choquant : ainsi des scènes supposées de viol chez Ronsard et Chénier par exemple ${ }^{86}$. Or, comme l'a montré Merlin-Kajman (2018) cette pratique, «qui n'est pas exactement civile, n'est pas non plus transitionnelle ${ }^{87}$. Dans le cas du poème ronsardien en cause (Amours, 1557, Sonnet 20), elle suspend « l'indétermination joueuse du texte » et porte atteinte à "son potentiel de plaisir inassignable » (ibid.). Accompagner l'événement de style dans le cadre d'une histoire du changement stylistique qui, attentive aux feuilletés interprétatifs, en préviendrait toute appropriation traumatique, relève d'une démarche assurément plus ouverte que celle, faite de stigmatisation et de censure, du « trigger warning ». Sans doute ne s'agit-il pas de réduire la non-additivité des causes, l'imprévisibilité de l'événement de style, mais d'en produire une explication probable, sans laquelle la transmission des productions lettrées risque de devenir de plus en plus verrouillée et de finir par s'abolir.

\section{BIBLIOGRAPHY}

Antoine, Gérald (1958-1962) : La Coordination en français, Paris, d'Artrey, t. 1 et 2.

Aron, Paul (2013) : « Le pastiche et la parodie, instruments de mesure des échanges littéraires internationaux », L. Gauvin, C. Van den Avenne, V. Corinus \& al. dirs, Littératures francophones. Parodies, pastiches, réécritures, ENS éditions, p. 23-42, https://books.openedition.org/enseditions/ 2437?lang=fr.

Badiou-Monferran, Claire (2011) : « Périodisation : le "français pré-classique" et l'“Early Modern French" ", Diachroniques, n 1, p. 83-109.

- (2013) : La littérarité des belles-lettres. Un défi pour les sciences du texte ? Paris, Classiques Garnier.

- (2017) : «L'"émergence" en sciences du langage : histoire et circulation d'un paradigme », A.-

M. Chabrolle-Cerretini dir., Paradigmes et concepts pour une histoire de la linguistique romane, Actes du colloque D.HI.CO.D.E.R (Dictionnaire Historique des COncepts Descriptifs de l'Entité Romane), Limoges, Lambert-Lucas,p. 57-66.

- (2018) : « De l'écrit oralisé à l'oral scripturalisé. L'évolution des emplois de et, jonctif de phrases et de propositions, dans les fictions narratives en prose des XVI ${ }^{\mathrm{e}}$ et XVII ${ }^{\mathrm{e}}$ siècles ", M.-G.

Lallemand et P. Mounier dirs, L'oralité dans le roman (XVIe et XVIIe siècles), Caen, Elseneur, $\mathrm{n}^{\circ} 32$, p. 53-74.

- (à paraître) : « Sémantique des coordonnants », Ch. Marchello-Nizia, B. Combettes, S. Prévost \&

T. Scheer dirs, Grande Grammaire Historique du français, Berlin, De Gruyter. 
Badiou-Monferran, Claire, dir. (2010) : Il était une fois l'interdisciplinarité. Approches discursives des Contes de Perrault, Louvain-la-Neuve, Academia Bruylant, coll. « Au cœur des textes ».

Badiou-Monferran, Claire \& Marchello-Nizia, Christiane (à paraître) : « Sémantique des connecteurs ", Ch. Marchello-Nizia, B. Combettes, S. Prévost \& T. Scheer dirs, Grande Grammaire Historique du français, Berlin, De Gruyter.

Badiou-Monferran Claire \& Capin, Daniéla (à paraître) : «Et "de relance” en diachronie longue : conjonction, connexion, interjection et marquage discursif ", A. Rodríguez Somolinos, S. Gómez Jordana Ferary \& M. Saiz Sánchez dirs, Actes du $12^{e}$ colloque du CILF. Marques d'oralité et représentation de l'oral en français, Chambéry, Presses de l'Université de Savoie.

Badiou-Monferran, Claire \& Monferran, Jean-Charles (à paraître) : «Et de relance dans les romans pantagrueliques : fait de langue ou fait de style?», P. Cifarelli \& F. Giacone dirs, Les Langues et les langages dans l'œuvre de François Rabelais, Genève, Droz.

Badiou-Monferran, Claire \& Ducos, Joëlle dirs (2012) : «Présentation », L'émergence : un concept opératoire pour les sciences du langage ?, L'Information grammaticale, $\mathrm{n}^{\circ} 134, \mathrm{p} .3-7$.

Benveniste, Emile (1966-1968) : Problèmes de linguistique générale, tomes 1 et 2, Paris, Gallimard.

Bordas, Éric (2005) : « Et la conjonction resta tensive. Sur le et de relance rythmique », Le Français moderne, $\mathrm{n}^{\circ} 73 / 1$, p. 23-39.

Buridant, Claude (1980a) : «Le strument "et” et ses rapports avec la ponctuation dans quelques textes médiévaux ", A.-M. Dessaux-Berthonneau dir., Théories linguistiques et traditions grammaticales, Lille, PUL, p. 13-53.

- (1980b) : « Les binômes synonymiques. Esquisse d'une histoire des couples de synonymes du Moyen Age au XVII ${ }^{\mathrm{e}}$ siècle », Bulletin du Centre d'Analyse du discours, nº 4, p. 5-79.

Capin, Daniéla \& Badiou-Monferran, Claire (à paraître) : « À l'aune de la transcatégorisation : modélisation des emplois de ET dans la langue d'hier et d'aujourd'hui », D. Th. Do-Hurinville dir., Transcatégorialité et transcatégorisation, Bulletin de la Société de Linguistique de Paris.

Citton, Yves (2007) : Lire, interpréter, actualiser. Pourquoi les études littéraires ? Paris, Éditions Amsterdam.

Cordingley, Anthony (2014), « L'oralité selon Henri Meschonnic », Palimpsestes, 27| 2014, mis en ligne le 01 décembre 2016, http://journals.openedition.org/palimpsestes/2029.

Denis, Delphine (2012) : “ "À la manière de" : le pastiche avant le pastiche », RHLF, n 112, p. 7-18, https://www.cairn.info/revue-d-histoire-litteraire-de-la-france-2012-1-page-7.htm? try_download=1.

Fagot-Largeault, Anne (2002) : «L'émergence », D. Andler, A. Fagot-Largeault et B. Saint-Sernin dirs, Philosophie des sciences II, Paris, Gallimard, p. 939-1049.

Ferland, Catherine (2007) : « Mémoires tabagiques. L'usage du tabac, du XVe siècle à nos jours ", Drogues, santé et société, Volume 6/1, p. 17-48, https://doi.org/10.7202/016942ar.

Fournier, Nathalie (2009 [2006]) : «L'ordre des mots en prose et en vers. Du Dom Juan de Molière au Festin de Pierre en vers de Thomas Corneille », F. Berlan \& al. dirs, Langue littéraire et changements linguistiques, Paris, PUPS, p. 315-334, <halshs-00387918>.

Genette, Gérard (1992 [1982]) : Palimpsestes : la littérature au second degré, Paris, Seuil. Gracq, Julien (1995 [1980]) : En lisant en écrivant, Euvres complètes, Paris, Gallimard, coll. « La Pléiade ». 
Grevisse, Maurice (1988 [1936], $12^{\mathrm{e}}$ rééd. Second tirage. Par A. Goosse) : Le Bon usage, Paris, Duculot.

Groupe de Fribourg (2012) : Grammaire de la période, Bern-Berlin-Bruxelles-Frankfurt am MainNew York-Oxford-Wien, P. Lang.

Jaubert, Anna (2007) : « La diagonale du style. Étapes d'une appropriation de la langue », Pratiques, $\mathrm{n}^{\circ} 135-136$, p. 47-62.

Kuhn, Thomas S. (2008 [1962], trad. fr. L. Meyer) : La structure des révolutions scientifiques, Paris, Flammarion, coll. «Champs ».

Kim, Jaegwon (2006 [2005], trad. fr. M. Mulcey) : Trois Essais sur l'émergence, Paris, Les éditions d'Ithaque.

Koch, Peter \& Esterreicher, Wulf (2001) : « Langage parlé et langage écrit », Lexikon der Romanistischen Linguistik, tome 1, Tübingen, Max Niemeyer Verlag, p. 584-627.

Llamas Pombo, Elena (2016) : « Ponctuation médiévale, pragmatique et énonciation. Lire l'Ovide Moralisé au XIV ${ }^{\mathrm{e}}$ siècle », Linx, $\mathrm{n}^{\circ}$ 73, 2, p. 113-146.

Marchello-Nizia, Christiane (1978) : « Ponctuation et "unités de lecture" dans les manuscrits médiévaux, ou : je ponctue, tu lis, il théorise ", Langue française, $n^{\circ} 40$, p. 32-44.

Ménager, Daniel (1989) : «La stratégie des titres dans Gargantua et Pantagruel », Cahiers Textuel, $1989, n^{\circ} 4 / 5$, p. 13-19.

Merlin-Kajman, Hélène (2018) : « Enseigner avec civilité ? Trigger warning et problèmes de partage en littérature », En ligne, site Mouvement-transitions, Rubrique littérarité, $n^{\circ} 4$.

- (2019) : « Encore Chénier, et au-delà », En ligne, site Mouvement-transitions, Rubrique Littérarité, $\mathrm{n}^{\circ} 7$.

Meschonnic, Henri (1999) : Poétique du traduire, Paris, Verdier.

- (2009 [1982]) : Critique du rythme. Anthropologie historique du langage, Paris, Verdier.

Molinié, Georges (2013) : « Penser la stylistique historique », C. Badiou-Monferran dir., La littérarité des belles-lettres. Un défi pour les sciences du texte? Paris, Classiques Garnier, p. 285-289.

Morel, Mary-Annick \& Danon-Boileau, Laurent (1998) : Grammaire de l'intonation, Paris, Ophrys.

Petitjean, André (1984) : « Pastiches et parodies. Enjeux théoriques et pédagogiques », Pratiques, $\mathrm{n}^{\circ} 42$, p. 3-33.

Philippe, Gilles (2000), « Les divergences énonciatives dans les récits de fiction », Langue française, $\mathrm{n}^{\circ} 128$, p. 30-51.

- (2005) : « Traitement stylistique et traitement idiolectal des singularités langagières ", Cahiers de praxématique, $\mathrm{n}^{\circ} 44$, en ligne, URL : http://praxematique.revues.org/1659.

- (2008) : « Registres, appareils formels et patrons », L. Gaudin-Bordes et G. Salvan dirs, Les registres. Enjeux stylistiques et visées pragmatiques, Louvain-La-Neuve, Academia-Bruylant, p. 27-37.

- (2013) Le Rêve du Style parfait, Paris, PUF.

- (2019, à paraître), « Sur l'émergence du présent romanesque », Poétique.

Philippe, Gilles \& Piat, Julien (2018) : « La langue littéraire à l'épreuve du temps », en ligne, Acta fabula, vol.19/1, http://www.fabula.org/acta/document10657.php. 
Philippe, Gilles \& Piat, Julien, dirs (2009), La langue littéraire. Une histoire de la prose en France de Gustave Flaubert à Claude Simon, Paris, Fayard.

Proust, Marcel, (1920) : « A propos du style de Flaubert », NRF, t. 4, Paris, Gallimard, p. 72-90.

Ripoll, Camille (2005) : « Systèmes dynamiques non-linéaires et concept d'émergence », J.

Guespin-Michel \& L. Sève dirs, Émergence, complexité et dialectique, Paris, Odile Jacob, p. 213-228.

Rossari, Corinne \& Cojocariu, Corina (2007) : « Parataxe et coordination », A. Rousseau dir., La Coordination, Rennes, Presses universitaires de Rennes, p. 127-139.

Serbat, Guy (1990) : «Et “jonctif” de proposition : une énonciation à double détente ", L'Information grammaticale, $\mathrm{n}^{\circ} 46, \mathrm{p} .26-28$.

Soutet, Olivier (2012) : «Effection et émergence en psychomécanique du langage », L'Information grammaticale, $\mathrm{n}^{\circ} 134$, p. 38-44.

Serres, Michel (1968) : Hermès 1, La communication, Paris, Minuit.

Thibaudet, Albert (1982 [1922/1935]), Gustave Flaubert, Paris, Gallimard, coll. « Tel ».

Vaugelas, Claude Favre de (2009 [1647]) : Remarques sur la langue françoise, éd. Z. Marzys, Genève, Droz.

Watine, Marie-Albane \& Yocaris, Ilias (2016) : « Le style comme événement. Appel à

communication », https://www.fabula.org/actualites/le-style-comme-evenement_78926.php.

Yocaris, Ilias (2016) : Style et semiosis littéraire, Paris, Classiques Garnier, coll. « investigations stylistiques ».

\section{NOTES}

1. Au sens où le style gagnerait à être pensé «non comme un "objet statique" ou une catégorie esthético-historique plus ou moins arbitrairement définie, mais comme un événement [voire "une suite ininterrompue d'événements (successifs et/ou simultanés)"] découlant de l'interaction entre les différentes composantes d'un texte donné (écrit ou oral, littéraire ou non), les genres et/ou les mouvements littéraires auxquels il fait éventuellement référence, l'instance réceptrice, le contexte énonciatif, le cadre sociologique dans lequel s'inscrit la démarche de l'énonciateur » (Yocaris 2016:24).

2. Parmi d'autres, sont également sollicités, quoique de manière plus ponctuelle, certains textes de Joinville, Ronsard, Malherbe, Laclos, Chénier, Zola, Proust, Céline...

3. Non que l'événement implique nécessairement un changement. L'événementialité stylistique peut bien évidemment s'éprouver et s'appréhender en dehors du cadre ici choisi.

4. Au sens large du terme, renvoyant à la période qui, conduisant de 1850 à 1990, correspond, selon Philippe \& Piat 2009, au moment d'autonomisation du champ littéraire (par rapport aux autres champs discursifs) et de sa langue (par rapport à la langue commune).

5. Empruntée aux travaux anglo-saxons et traduite en français par « Première modernité ", cette dénomination renvoie à la période conduisant du XVIe s. au XVIIIe s. Sur les enjeux du choix terminologique opposant « Première modernité » et « (Pré-)classicisme », voir Badiou-Monferran (2011).

6. Dans le prolongement des travaux de linguistique de Badiou-Monferran \& Ducos (éds, 2012) sur le concept d'émergence en sciences du langage, Philippe interroge, dans "L'émergence du présent romanesque» (à paraître en 2019), les conditions de possibilité d'une histoire 
émergentiste $\mathrm{du}$ changement stylistique, en valorisant, parmi les nombreux critères définitoires de l'émergence, celui du « saut qualitatif ».

7. Voir Morel \& Danon-Boileau (1998).

8. Tous les exemples non référencés sont empruntés aux éditions de la base Frantext. Sauf exception, tous les soulignements nous reviennent.

9. Dans ce cas, et est paraphrasable par « enfin » : «Pourtant mon amy dictes moy qui estes vous, dont venez vous, où allez vous, que querez vous / et quel est vostre nom ? » (Rabelais, Pantagruel, 1532, Lyon, Claude Nourry, chap. 9, p. 49. Le signe / correspond à une virgule). Il est compatible avec enfin, finalement (et autres connecteurs conclusifs apparentés) : "A-t-elle eu sa vie volée, Mother? Oui et non, comme tout le monde. Et finalement, plutôt non, c'est le sens du message ultime [...]» (Sollers, Le Secret, 1993 : 129).

10. Le cas échéant, et est paraphrasable par "si bien que »: " mais la faulse vieille vesnoit et vessoit puant comme cent diables / \& le pouvre regnard estoit bien mal a son ayse » (Rabelais, Pantagruel, 1532, Lyon, Claude Nourry, chap. 11, p. 86). Il est compatible avec en conséquence (et autres connecteurs consécutifs apparentés) : «Être à venir de la conscience, il n'est plus, de ce fait, de la conscience. Et, en conséquence, il est bien totalement relatif à elle » (Sartre, Carnets de la drôle de guerre, 1939-1940, 1983 : 395. Premier soulignement de Sartre. Second soulignement de nous).

11. Dans ce cas, et est paraphrasable par « mais » : « Tu es Lymousin pour tout potaige. Et tu veulx icy contrefaire le Parisien » (Rabelais, Pantagruel, 1532, Lyon, Claude Nourry, chap. 6, p. 34). Il est compatible avec cependant (et autres connecteurs oppositifs apparentés) : «Il se sentait la figure toute rouge. Et cependant, il était délicieusement bien » (Guérin, L'Apprenti, 1946 : 92).

12. Du moins si l'on suit Rossari \& Cojocariu (2007), qui contestent la lecture « conditionnelle» traditionnellement faite des constructions en question. Et est (selon Rossari \& Cojocariu 2007) paraphrasable par "pour que », moyennant une transformation des mode et temps verbaux du second conjoint : «Aydez-vous seulement, et Dieu vous aydera (Régnier, Les Satires, 1603, 13, p. 117). Il est compatible avec alors : «Donnez-moi le temps de vous connaître un peu plus, et alors je me permettrai peut-être de discuter quelquefois avec vous » (Sand, Mademoiselle de la Quintinie, $1877: 31)$.

13. Dans la présente contribution, afin d'illustrer notre propos, nous ne nous intéresserons presqu'exclusivement qu'aux enchaînements de type phrastique et/ou périodique. Pour une histoire de l'ensemble de ces tours par "relance", à tous les niveaux, syntagmatiques, propositionnels, phrastiques et/ou périodiques, voir Badiou-Monferran (à paraître).

14. Et à l'articulation d'une succession temporelle constitue un cas intermédiaire situé entre la clôture et la relance. Possiblement co-occurrent avec les adverbiaux puis, alors, après, ensuite [...], il vient spécifier un lien implicatif entre un avant et un après et est, à ce titre, difficilement supprimable, tout comme n'importe quel et de clôture. En revanche, tout comme n'importe quel et de relance, il peut se démultiplier, comme dans l'exemple suivant : «Julien soupira. - Les cris. En allemand. Très loin. Et puis, un coup de fusil. Et ensuite une rafale...Et puis plus rien. Il dut se taire. Sa salive ne passait plus dans sa gorge serrée. » (Clavel, Le cour des vivants, 1964 : 121). On notera que lorsque et, comme dans l'exemple ci-dessus, est co-occurrent avec un adverbial temporel (puis, ensuite...) se chargeant d'expliciter le lien implicatif entre l'avant et l'après, il redevient optionnel et fonctionne, dans le cadre de cette combinaison, comme un marqueur discursif à part entière (voir la note ci-dessous), venu « relancer » l'énonciation en cours.

15. Les linguistes utilisent tantôt le terme de «marqueur discursif» (entre autres, Schiffrin 1987 ; Dostie 2004; Dostie \& Pusch 2007; Dostie \& Lefeuvre 2017) tantôt celui de «marqueur pragmatique " (parmi d'autres, Gonzalès 2005: 53; Brinton 2008: 214; Aijmer et SimonVandenbergen 2011 : 223-247). Indépendamment de la terminologie adoptée, lesdits marqueurs «se caractérisent par (i) leur optionalité syntaxique ; (ii) leur position très souvent initiale (iii) leur réduction phonologique (iv) leur capacité à articuler les plans discursifs local et global (v) 
leur sous-détermination sémantique (vi) enfin, leur fonction cohésive [... et leur capacité à ] signaler un positionnement énonciatif et/ou interactionnel » (Badiou-Monferran \& MarchelloNizia, à paraître).

16. Voir également Marchello-Nizia (1978) et de Buridant (1980a, 1980b).

17. Exemple emprunté à Badiou-Monferran \& Capin (à paraître), et traduit du français médiéval par Daniéla Capin, que nous remercions. Le signe $\mathrm{Z}$ renvoie à la note tironienne abrégeant le et (aussi noté $e$ ) dans les manuscrits français médiévaux.

18. «Le et qui met en relation des segments de fonction équivalente acquiert le statut de connecteur pragmatique argumentatif grâce à une ponctuation marquée, qui infère une relation d'insistance ou de relief sur la co-orientation de deux arguments. Cette valeur marquée reste soulignée par l'emploi [...] du punctus planus, le simple point [...] en alternance avec [...] la virgula. Ces deux signes ajoutent une valeur pragmatique à la particule et [...]» (Llamas Pombo 2016 : 131).

19. Dans les productions imprimées, l'association d'un ponctuant et du MP et devient monnaie courante et est attestée dès le XVI siècle pour tous types d'enchaînements, même ceux dans lesquels et, en cooccurrence avec un adverbial comme puis ou ensuite, ne hiérarchise pas les conjoints et se contente de spécifier une relation de successivité temporelle. Voir parmi d'autres l'édition de 1532 de Pantagruel (où le signe «/ " correspond à l'actuelle virgule) : «Adoncques Panurge mist le doigt indice de la dextre dedans la bouche/ le serrant bien fort avecques les muscles de la bouche / et puis le tiroit [...]» (Rabelais, Pantagruel, 1532, Lyon, Claude Nourry, chap. 14 : 81-82 [site BVH]) ; « A quoy Panurge baissa sa teste du couste gauche \& mist le doigt meillieu en l'oreille dextre/ elevant le poulce contremont. Et puis croisa les deux bras sur la poictrine [...]» (ibid. : 82).

20. A propos de la combinaison « et puis » se chargeant de marquer une succession temporelle, voire la note 14 .

21. Transcription de Morel \& Danon-Boileau (1998), citée par le Groupe de Fribourg (2012:150).

22. Il existe bien entendu des exceptions. Ainsi, dans le roman La Citadelle de Saint-Exupéry (1944), les suites en [A. Et B] représentent jusqu'à $23 \%$ du total des enchaînements après un point (2207 occurrences sur 9628), contre 12,4\% pour l'ensemble de la production romanesque enregistrée pour l'année 1944 dans la base Frantext (2534 occurrences sur 20409), et 6\% pour le total des productions (romanesques et non romanesques) de l'année 1944 de la base (2971 occurrences de [A. Et B] sur 50143 enchaînements après un point).

23. Dans l'argumentaire du présent numéro, Watine \& Yocaris (2016) définissent «l'événement de style " sur la base des cinq critères suivants : imprévisibilité ; sur-signification ; rupture ; intensité ; adresse.

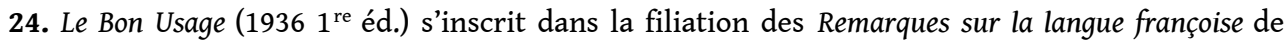
Vaugelas (1647). Ces dernières portent, comme on le sait, sur les usages - et parmi eux, sur le bon usage - du français. Par son titre, la somme de Grevisse leur rend ainsi hommage.

25. Romano (1998: 62), cité dans l'argumentaire de la présente livraison.

26. À la suite de Philippe (2008), le "patron" peut être défini comme un ensemble de traits langagiers convenus et désémantisés, se contentant de faire signe en direction d'un modèle linguistique propre à l'imaginaire d'une époque : « Dans l'histoire des formes littéraires, certains faisceaux de formes tendent [...] à se stabiliser, puis à figer, et donc à se désémantiser, du moins partiellement. Nous proposons d'appeler "patrons" le résultat d'un tel processus. L'apparition de ces patrons est moins exigée par les nécessités expressives de tel ou tel régime discursif que par une forme de conventionnement dans l'imaginaire langagier d'une époque: disons, pour aller vite, que les "appareils formels" regroupent des faits langagiers sémantiquement congruents, tandis que les "patrons" regroupent des faits langagiers sémiotiquement congruents. Le "patron oral" sera ainsi constitué de marquages hétéroclites sans cohérence dénotative [...] mais qui 
permettent à la prose écrite de prendre des allures “orales", selon la stéréotypie en vigueur au moment de la production du texte» (Philippe $2008: 31$ ).

27. Ces formulations nous reviennent.

28. Voir le chapitre 1 de Philippe \& Piat (2009, «Langue littéraire et langue parlée » : 57-89).

29. "Par "divergences énonciatives", on entendra ici les faits de manquement, au moins apparent, à la règle de cohérence énonciative dans la formation des énoncés : il y a divergence dès lors qu'un énoncé propose des marquages énonciatifs qui ne semblent pas pouvoir être assumés par un seul valideur, ou ne pas pouvoir être validés sur le même plan énonciatif » (Philippe $2000: 31$ ).

30. Pour un panorama exhaustif, voir Philippe \& Piat 2009, chap. 1 .

31. Nous empruntons cette citation à Philippe (2013:200).

32. Philippe (2013: 190), à qui nous empruntons cette citation, commente le pastiche perecquien en ces termes: "c'est bien cela: rythme anapestique (phrase courte, phrase courte, phrase longue), ouverture du dernier segment par et, suspension immédiate de la relance par un insert participial. Pas de doute. C'est du Flaubert. »

33. Cité dans Bordas (2005: 30, n. 22), qui commente ce passage en ces termes: «Zola [...] a souvent utilisé [le et de relance] comme marqueur de parodie [...]. Le parallélisme posé par la polysyndète de ce et biblique, par l'égalité prosodique des deux phrases (10-5// 10-7), le topos romantique trivialisé : tout suggère [une reprise parodique] de Madame Bovary. »

34. Au sens où «l'oral est compris comme un primat du rythme et de la prosodie dans l'énonciation » (Meschonnic 1999 : 117).

35. Apollinaire, "Jean Royère ", paru dans La Phalange et repris dans Euvres en prose, La Pléiade, 1992 (éd. M. Décaudin).

36. Voir parmi d'autres Pommier, Assez décodé, «Un vers très sollicité ", http:// rene.pommier.free.fr/Chantre.htm.

37. Comme le suggèrent fort justement les deux coordinateurs du présent numéro, dans le cas d'Apollinaire, il serait bien entendu toujours possible de rapporter rétrospectivement le Et en question à la poétique de la surprise que le poète appelle de ses vœux quelques années plus tard : «La surprise est le grand ressort nouveau. C'est par la surprise, par la place importante qu'il fait à la surprise que l'esprit nouveau se distingue de tous les mouvements artistiques et littéraires qui l'ont précédé » («Conférence du 26 Novembre 1917 », Paris, Théâtre du Vieux-Colombier. Les italiques sont d'Apollinaire. La citation nous a été communiquée par Yocaris et Watine, que nous remercions). Pour autant, dans la mesure où le procédé n'est pas récurrent, rien ne permet de le rattacher avec certitude au faisceau de déterminations langagières venu sous-tendre, de façon stable et concertée, l'effet de surprise apollinarien. Si le Et initial du poème d'Alcools rencontre ce dernier, peut-être s'agit-il là d'un heureux hasard.

38. Dans l'ensemble de cette contribution, nous renvoyons pour les références à l'édition du Pantagruel de 1532 aux pages de l'édition Saulnier (Pantagruel, éd. V. L. Saulnier, Genève, Droz, 1965), tout en restituant le texte dans son orthographe et sa ponctuation originale, d'après l'édition Nourry retranscrite sur le site des Bibliothèques virtuelles Humanistes. Pour toute référence aux autres éditions de Pantagruel ou à d'autres livres de Rabelais, nous renvoyons à l'édition Huchon (Euvres complètes, éd. M. Huchon, Paris, Gallimard, 1994) et à son appareil de variantes.

39. Sur cette question, voir les relevés de Jacques-Charles Brunet, Recherches bibliographiques et critiques sur les éditions originales des cinq livres du roman satirique de Rabelais et sur les différences de texte qui se font remarquer particulièrement dans le premier livre du Pantagruel et dans le Gargantua, Paris, Potier, 1852, notamment p. 46-47; voir également certains éléments de commentaires chez Gérard Defaux à propos des remaniements de 1534 dans Pantagruel et les sophistes, La Haye, Nijhoff, 1973, p. 6-13 ; voir enfin Mireille Huchon, Rabelais grammairien, De l'histoire du texte aux 
problèmes d'authenticité, Genève, Droz, 1981. Toutes les données concernant et de relance sont détaillées dans Badiou-Monferran \& Monferran (à paraître).

40. Il y a 19 suppressions dans l'édition de 1542. Voir p. 231, var. l; p. 266, var. p; p. 269, var. $n$; p. 272 , var. $h$; p. 273 , var. $b$; p. 281, var. $d$; p. 288 , var. $l$; p. 293, var. $k$ et $m$; p. 305 , var. $d$ et $k$; p. 306 , var. $n ;$ p. 321, var. $f$ et $o ;$ p. 330, var. $g$; p. 335, var. f. Rappelons enfin qu'après examen, le fait que et soit transcrit ou non par l'esperluette ne semble avoir aucune incidence sur la disparition de la forme.

41. Voir Badiou-Monferran \& Monferran (à paraître).

42. A la suite de Yocaris \& Watine (2016), rappelons que dans l'argumentaire du présent numéro, «l'intensification» qui accompagne souvent la perception de l'événement se définit moins en termes de fréquence qu'en termes d'impact émotionnel ou esthétique. Pour autant, comme les deux processus ne sont pas symétriques, la suppression massive des et de relance laisse à penser que ceux-ci n'étaient pas perçus comme des vecteurs d'intensité et/ou que, s'ils l'étaient, leur suppression a contribué à la dés-intensification des textes et discours qui, initialement, les contenaient.

43. Avec toutefois, du français renaissant au français classique, une diminution importante de leur empreinte de fréquence (voir ci-dessus, le point 1).

44. Si tant est que ceux-ci constituent un récit condensé. Voir les titres des chapitres XIII, XIV, XV, XVII, XX, XXI, XXII et XXIII, et à ce propos Ménager (1989).

45. On ne trouvera dans ces pages que des et marqueurs d'interjection. En voici la liste: Et ou diable sont ils allez? (chap. XII : 98) ; Et quelz proces as tu peu avoir ? disoys -je (XII : 99 ) ; Et à quelle fin? dis je. (XII : 101); Et a quoy ny comment? dys je (XXII : 171); Jesus (dys je) et ou? (XXII : 172). Ces configurations illustrent bien un cas distinct de celui de et de relance, tout du moins, un cas particulier où et de relance devient candidat à la réanalyse. Badiou-Monferran \& Monferran (à paraitre) rappellent en effet que, notamment au XVIe siècle, et dans ces emplois est perçu non pas comme un connecteur, mais comme un marqueur d'affectivité, en vertu de son homophonie avec l'interjection (hé!, eh !), ainsi qu'en témoigne Henri Estienne (« La conjonction françoise $E t$ s'accorde très bien avec la Grecque kai, en une signification extraordinaire, telle que par ci-devant nous avons observé aux Adverbes eita et epeita, quand nous disions qu'ils emportoyent une declaration de despit ou indignation. » Henri Estienne, Traicté de la conformité du langage françois avec le grec, 1569, cité par Gérald Antoine 1962 : 930).

46. Koch \& Esterreicher (2001 : 584-627).

47. Voir à ce sujet Fournier (2009: 1) : "On connait l'histoire mouvementée du Dom Juan de Molière; étouffée au théâtre du vivant de Molière, puisqu'elle est créée le 15 février 1665 et retirée après la relâche de Pâques, la pièce est également étouffée en librairie : le privilège de 1665 n'est pas utilisé et la pièce est publiée pour la première fois dans l'édition [...] de 1682, édition dite " cartonnée ", mélange d'autocensure et de censure, qui sera la seule version connue de Dom Juan tout au long des XVIIIe et XIXe siècles. Il faut attendre 1819 pour que le texte intégral de la pièce soit établi par l'éditeur Auger, et 1841 pour que le Dom Juan authentique de Molière remonte sur la scène [...] Entre temps, la pièce de Molière aura été remplacée, au théâtre et en librairie, par le Festin de Pierre de Thomas Corneille, comédie en vers composée à la demande d'Armande Béjart, créée avec un vif succès en février 1677 et imprimée en mars 1683. Et c'est cette version du Dom Juan, toujours présentée comme l'œuvre de Molière, qui sera jouée pendant presque deux siècles, jusqu'à ce que la résurrection du vrai Dom Juan en prose la fasse tomber à son tour dans l'oubli ».

48. Nous nous fondons sur la version du texte de 1682 présentée sur le site Molière 21 : (http:// moliere.huma-num.fr/analyse_textes.php).

49. Comme rappelé sous 1 , dans la seconde moitié du XVIIe siècle, la fréquence des et de relance à l'écrit chute globalement, notamment en énonciation historique, et le tour n'apparait plus guère qu'en énonciation de discours. 
50. Fournier (2009) emprunte la terminologie de "préambule» et de "rhème » à Morel et Danon-Boileau (1998), qui les ont conçus pour décrire l'organisation des énoncés de l'oral spontané du XXe siècle.

51. Voir ci-dessus la note 47.

52. Sur la différence de fonctionnement entre l'emploi de marqueur pragmatique et celui de connecteur, voir la section 1 et sa note 9 .

53. Première occurrence de et jonctif de proposition, en emploi de MP.

54. Deuxième occurrence de et jonctif de proposition, là encore en emploi de MP.

55. Troisième occurrence de et jonctif de proposition, cette fois en emploi de connecteur temporel.

56. Quatrième occurrence de et jonctif de proposition, en emploi de connecteur consécutif.

57. Cinquième occurrence de et jonctif de proposition, là encore en emploi de connecteur consécutif.

58. Sixième occurrence de et jonctif de proposition, en emploi de connecteur conclusif.

59. Au sens où il peut fonctionner, suivant les contextes, soit comme un coordonnant, soit comme un connecteur, soit comme un marqueur pragmatique (voir Capin \& Badiou-Monferran, à paraître).

60. Voir Badiou-Monferran \& Capin (à paraître). Et coordonnant est (i) obligatoire (ii) associe des conjoints réversibles (« Un et deux font trois ») ; et connecteur est (i) obligatoire (ii) et associe des conjoints irréversibles; et marqueur pragmatique est (i) facultatif (ii) et associe des conjoints irréversibles.

61. Dans le cadre d'une stylistique auteuriste, on trouvera bien entendu des exceptions. Parmi d'autres, voir ici-même la contribution de Nicolas Laurent sur Pascal.

62. Voir à ce sujet le collectif de Feltz, Crommelinck \& Goujon dirs (1999), Auto-organisation et émergence dans les sciences de la vie.

63. Parmi d'autres, voir, «Émergence. La théorie qui bouscule la physique », La Recherche, 405, 2007.

64. Sur l'histoire et la circulation du paradigme émergentiste en sciences du langage, voir Badiou-Monferran 2017.

65. Nous reprenons ici pour partie des considérations exposées dans Badiou-Monferran 2017.

66. Dans Badiou-Monferran \& Ducos dirs (2012), Nyckees (2012:134) illustre ce trait définitoire avec l'exemple de la construction de la termitière : "chacun des membres de la colonie ne vise qu'à déposer sa charge à un endroit choisi en fonction d'un gradient olfactif, mais tous ensemble, sans rechercher un tel effet, édifient une orgueilleuse cathédrale gothique ». Autrement dit, la cathédrale n'était pas contenue dans les poutres d'où sont issues les charges des termites.

67. Sur ces quatre points, voir Fagot-Largeault (2002). Pour une synthèse des rapports entre le paradigme émergentiste et i) la théorie du chaos, ii) la théorie de la complexité, iii) la théorie des systèmes dynamiques non linéaires, voir Guespin-Michel \& Sève dirs (2005). Pour une discussion de la notion d'émergence, voir Kim (2006).

68. Voir Aron (2013: 23), qui y intègre « des productions diverses, du plagiat à la charge, de la supposition d'auteur à la satire", en soulignant que les plus connues sont «le pastiche et la parodie ». Celle-ci en appelle à un processus de "transformation », celui-là à un processus d'« emprunt ».

69. Philippe (2013) montre bien que l'un ni l'autre de ces deux écrivains ne revendiquent au demeurant le style de Flaubert comme modèle d'écriture à part entière, et encore moins pour eux-mêmes. L'un et l'autre pratiquent le pastiche comme malgré eux. Voir Perec (1965) : «Je me suis senti un peu ligoté [...]» (cité dans Philippe $2013: 190$ ), et Cocteau (1947) : « le style que je réprouve, dont celui de Flaubert est le type » (cité dans Philippe 2013 : 195). 
70. Nous empruntons cette expression à Philippe (2005: 85), qui oppose deux traditions stylistiques : celle germanique, de la « stylistique des styles »; celle, française de la « linguistique des styles".

71. Ceux où le locuteur se voit contraint d'exhiber son identité langagière : cas qui intéressent plus, au demeurant, la pragmatique des genres du discours que la stylistique (voir Philippe, 2005 : $78 ; 88$, n. 2).

72. Une tradition qui remonte à Buffon (« le style est l'homme même »), et qu'ont entre autres ralliée, par des voies différentes, Bruneau d'abord (1951), puis Rastier (1994; 2001). Voir à ce sujet Philippe 2005.

73. Voir Denis (2012).

74. Par-delà l'argumentaire du présent numéro, voir également la rubrique « intensités » du site

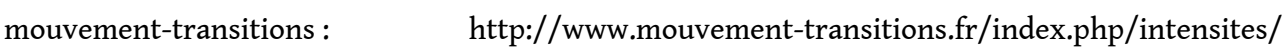
presentation, qui, dans sa " Présentation ", met en avant, tout à la fois, le caractère inhabituel de ce type d'interrogation, et la nécessité de se saisir de cette question : «Intituler une rubrique de recherche "Intensités" n'est pas habituel. Nous désirons rappeler les chercheurs à une certaine forme d'engagement, les inviter à se risquer, à écarter un peu les barrières protectrices de leur spécialisation [...]».

75. Selon les termes de Watine \& Yocaris 2016, dans l'argumentaire de la présente livraison.

76. Au sens où l'entendent Watine \& Yocaris 2016 dans l'argumentaire du numéro : « Dans une approche événementielle, le style [...] relève-t-il uniquement de la manière dont le lecteur appréhende les composantes textuelles, en les inscrivant dans une durée temporelle (cf. Jaubert 1990, 2007) et en choisissant de les rattacher à des valeurs stylistiques de toutes sortes (cf. Yocaris 2016) ?»

77. Au sens où l'émergence désigne un «cas particulier de système complexe » dont «l'autoorganisation » repose sur des modulations dues aux interactions de «variations [...] internes au systèmes » et de « contraintes externes » (Badiou-Monferran \& Ducos $2012: 3$ ).

78. Mais ce, dans les limites de ce que à quoi peut prétendre ce type d'entreprise. Voir à ce sujet Merlin Kajman (dans Badiou-Monferran dir. 2010 : 229) : « Le contexte exhaustif reconstruit par un chercheur ne correspond à rien historiquement. Il n'y a pas de lecteur au savoir aussi complet que le chercheur [...]. Reconstruire un contexte ne permet pas de parvenir enfin au niveau de certitude et de positivité factuelle escompté. Quand on lit les polémiques [ie, les Querelles littéraires du XVII ${ }^{\mathrm{e}}$ siècle], on s'aperçoit à quel point les contemporains s'accusent mutuellement de ne rien comprendre. »

79. C'est-à-dire, la somme des lectures qui ont succédé, historiquement, à celle(s) du contexte de production, et qui, en diachronie, ont contribué, tout à la fois, à la transmission et à l'actualisation du texte/discours. Molinié (dans Badiou-Monferran dir. 2013 : 286), oppose la « réception impliquée » et la "réception d'archive» en ces termes : «Dans ce cas-ci, [...] la réception construit un travail d'interprétation, qui correspond à la posture du critique, appuyé sur de la documentation socio-historique de toute nature. Dans ce cas-là, [...] le travail d'interprétation] intègre l'affectivité de chaque lect[eur] dans une histoire au présent à chaque acte de réception [...] Au bout du compte, le critique, l'analyste, argumente un feuilleté de réception [...]».

80. Voir parmi d'autres Citton (2007).

81. Voir ici-même, dans l'argumentaire de la présente livraison : "Adresse : l'événement est ce qui arrive à quelqu'un, sollicite son attention, ses ressources cognitives; au-delà, il l'institue comme un sujet particulier, celui à qui arrive l'événement, et qui y répond - voire même celui qui advient par l'événement. »

82. Marel \& Marel (1987 : 33) : Molière, Dom Juan, Paris, Bordas, coll. « Univers des Lettres », Texte intégral.

83. Voir notre note 26 . 
84. Yocaris (2016:24).

85. «Avertissement qui prévient qu'une œuvre contient des éléments pouvant déclencher le rappel d'un traumatisme " (Wikipédia).

86. Au sens où elle verrouille autoritairement l'interprétation alors que les textes littéraires sont par essence polysémiques et constituent, à ce titre, un espace de jeu, créatif, libre, libéré par làmême de toute tension traumatique, au même titre que l'aire transitionnelle du nourrisson chez Winnicott. Concernant le cas Ronsard, voir Merlin-Kajman (2018) : http://www.mouvementtransitions.fr/index.php/litterarite/articles/n-4-h-merlin-kajman-enseigner-avec-civilite-

trigger-warning-et-problemes-de-partage-de-la-litterature. Concernant le cas Chénier, voir Merlin-Kajman (2019): http://www.mouvement-transitions.fr/index.php/litterarite/articles/ n-7-h-merlin-kajman-encore-chenier-et-au-dela.

87. Au sens où elle ne permet pas de réguler les rapports des individus au monde.

\section{ABSTRACTS}

En prenant pour observatoire l'entrée formelle des et « de relance ", constamment sollicités par les écrivains français de Rabelais à Duras, cette contribution se propose d'éprouver la validité (descriptive et explicative) de la proposition d'« événement de style » en diachronie. Elle montre comment cette modélisation, élaborée par Yocaris (2016) et Watine et Yocaris (2019) pour et à partir des fictions post-modernes, rencontre des difficultés à décrire les corpus de l'ancien régime littéraire (16e-18e siècles), soumis à une logique moins "événementielle " que «variationniste». Dans la perspective d'une histoire du changement stylistique tout à la fois discrète et continuée, elle interroge alors la capacité du modèle de l'« émergence " (BadiouMonferran et Ducos 2012), à prendre en charge l'entier de cette histoire, et à penser l' « événementialisation » de la langue littéraire des Belles-Lettres.

\section{INDEX}

Chronological index: XVIe siècle, XXIe siècle

Mots-clés: événement, variation, émergence, diachronie, « et »

\section{AUTHOR}

\section{CLAIRE BADIOU-MONFERRAN}

Université de Sorbonne Nouvelle - Paris 3, EA 7345 CLESHIA - Langage, Systèmes, Discours Claire Badiou-Monferran est professeur de stylistique françaises à l' Université de Sorbonne Nouvelle - Paris 3. Elle est spécialiste du français classique et de ses modèles hérités dans la prose littéraire moderne et contemporaine. Ses travaux relèvent essentiellement de la linguistique diachronique et de l'histoire du changement stylistique. Elle a notamment publié un ouvrage sur La Bruyère (Les conjonctions de coordination ou « l'art de lier ses pensées » dans les Caractères, Paris, Champion, 2000), et plusieurs collectifs (dont, en collaboration avec J. Ducos, L'émergence : un concept opératoire pour les sciences du langage? L'Information Grammaticale $\mathrm{n}^{\circ} 134,2012$, et, à titre de 
seul éditeur, La littérarité des Belles-Lettres. Un défi pour les sciences du texte?, Classiques Garnier, 2013). Elle coordonne actuellement un numéro sur la notion de « rémanence » en sciences du langage (à paraître dans Le français moderne, en 2020). 